\title{
Selectivity on-target of bromodomain chemical probes by structure-guided medicinal chemistry and chemical biology
}

Targeting epigenetic proteins is a rapidly growing area for medicinal chemistry and drug discovery. Recent years have seen an explosion of interest in developing small molecules binding to bromodomains, the readers of acetyl-lysine modifications. A plethora of co-crystal structures has motivated focused fragment-based design and optimization programs within both industry and academia. These efforts have yielded several compounds entering the clinic, and many more are increasingly being used as chemical probes to interrogate bromodomain biology. High selectivity of chemical probes is necessary to ensure biological activity is due to an on-target effect. Here, we review the state-of-the-art of bromodomain-targeting compounds, focusing on the structural basis for their on-target selectivity or lack thereof. We also highlight chemical biology approaches to enhance on-target selectivity.

First draft submitted: 9 March 2016; Accepted for publication: 22 April 2016; Published online: 19 May 2016

Keywords: BET bromodomains • chemical genetics • chemical probes $\bullet$ selectivity - structure-based drug discovery

Many physiological and pathological cellular processes are regulated by epigenetic mechanisms as a response to environmental stimuli [1]. It is well known that epigenetic regulation is controlled by chemical modifications on DNA and on post-translational modifications (PTMs) on histones, including acetylation, methylation and phosphorylation $[2,3]$. These chemical modifications on the histone amino acids are recognized by specific multidomain proteins able to write, read or erase them, and deregulation of these processes has been associated to disease [4-6]. Cancer was the first human disease linked to epigenetics. In 1983, Feinberg et al. found that genes of colorectal cancer cells were hypomethylated compared with normal tissues [7]. Since then, efforts have been devoted to increase knowledge in epigenetics and in particular to exploit understanding of these processes in order to develop new targeted molecular therapeutics $[8,9]$.
Acetylation of the $\varepsilon$-amino group of lysine residues (KAc) is one of the most common modifications of histone tails [10,11]. Acetylation levels are reversibly maintained by histone acetyltransferases (HAT) and histone deacetylases (HDAC) that respectively write and erase this PTM [12]. HATs and HDACs are often deregulated in diseases through mechanisms that include aberrant expression levels, the occurrence of mutations as well as truncations, and chromosomal rearrangements [13]. From a drug discovery point of view, to date only a very limited number of HAT inhibitors have been described and the investigation of HAT inhibitors has been mostly limited to in vitro studies of growth inhibition of cell lines [14]. In contrast, several small molecules able to inhibit HDAC catalytic activity have been discovered and have also entered clinical trials with five examples already approved [15]. HDACs are to date
Carles Galdeano ${ }^{1,2}$ \& Alessio Ciulli*,1

'Division of Biological Chemistry \& Drug Discovery, School of Life Sciences,

University of Dundee, James Black Centre, Dow Street, Dundee, DD1 5EH, UK

${ }^{2}$ Institut de Biomedicina de la Universitat de Barcelona (IBUB) \& Departament de Fisicoquímica, Facultat de Farmàcia, Universitat de Barcelona, Av. Joan XXIII s/n, 08028 Barcelona, Spain *Author for correspondence: Tel.: +44 1382386230 Fax: +44 1382386373 a.ciulli@dundee.ac.uk 
the most explored epigenetic drug target family by ligand efficiency hits from fragment screening camthe phast experis In contrast, much less has been known of the read- paigns that could be really optimized for potency. In ing process of acetylation marks in histones, and tar- in drug discovery programs, these new molecules geting protein interactions mediated by epigenetic could be used to elucidate the biological function of readers of this PTM had remained largely unexplored bromodomains and their importance as therapeuuntil recently. However, this suddenly changed in 2010 tic targets, in other words, as high-quality epigenetic with the publication of potent and selective triazolo- chemical probes [20,21]. However, the high structural diazepine-based inhibitors of Bromo and Extra-Ter- conservation of bromodomains pose a significant chalminal domain (BET) proteins, (+)-JQ1 and I-BET762 lenge toward developing suitable single-target selective (Figure 1) [17,18], which were shown to have in vivo on- inhibitors. Lack of selectivity poses a potential limitaarget activity in models or NUT midine carcinoma tion to bromodonain inhibitors as chencical probes as and inflammation, respectively. BET inhibitors bound it may confound the association of the cellular activity to highly conserved regions of BET proteins, called of a given probe to a particular bromodomain target. bromodomains, which were known to recognize the This has spurred mounting interest in developing more KAc modification in histones and other substrates. These two groundbreaking discoveries demon作 KAc in Kent fforits anc $x$ exple an explosion of small nolecules discoved or designed ily but also from other brinodomain classes, as well domains in the human phylogentic tree. This has in domins asf oftarets for example, of protein kinase prose

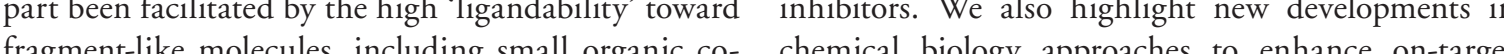
solvents such as DMSO and NMP (19, This propen- selectivity of brom apporat

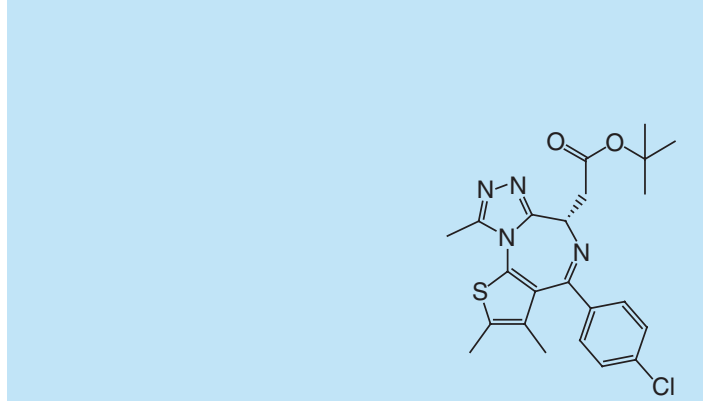

(+)-JQ

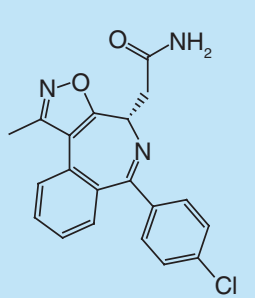

CPI-0610

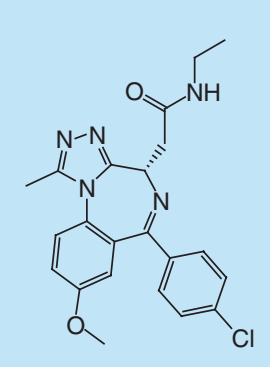

(GSK525762)
Figure 1. BET bromodomain inhibitors. Representation of the chemical structure of (+)-JQ1, I-BET762, CPI-0610,

Figure 1. BET bromodoma
Bromodomains \& bromodomain-containing proteins: structure, inhibition by chemical probes \& emerging role as drug targets

Bromodomains are an evolutionarily conserved family of $\sim 10$ amino acid modules found in histone acetyl and transcriptional regulators [22]. The human genome encodes 46 of such bromodomain-containing protein (BCPs) [23]. Each of the 46 proteins contains one to six bromodomains, giving a total number of 61 unique individual human bromodomain sequences. Based on sequence similarity, the whole human family of bromodomains can be divided into eight diverse subfamilies (group I-VIII) each containing at least thre bromodomains and comprising proteins of diverse functions [24]. While most $\mathrm{BCPs}$ recognize KAc marks on histone tails, evidence suggests that bromodomains
also are able to bind to acetylated proteins beyond also are able to bind to acetylated proteins beyon histones [19]. An example of PTM recognition in nonhistone substrate is the HIV-1 Tat, which gets acetyPCAF vosition $\mathrm{K}$. and this allows it to associate

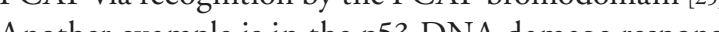
signal cascade, ignal casce wit CREBBP via its brom of the a dinate activation CR Despite their low overall bromodomains share a conserved fold comprising a bundle of four $\alpha$-helices (named $\alpha Z, \alpha A, \alpha B$ and $\alpha C$ ) linked by more diverse loop regions (Figure 2A). Two of these loops (ZA and $\mathrm{BC}$ ) that make up the mouth of the KAc binding site show large sequence variation and contribute to substrate specificity [28,29]. Crystal structures with bound histone and substrate peptides have shown that KAc is recognized within a hydrophobic pocket and anchored by forming a conserved hydrogen bond between its side chain amide group and the side chain amide of a highly conserved Asn residue [30]. In addition, a water-mediated hydrogen Tyr residue. Both residues are located at the botton of the ZA loop of bromodomains (Figure 2B) [31]. Another characteristic feature at the base of the KA recognition pocket is the presence of a network of water molecules that forms hydrogen bonds with the carbonyl group of the substrate KAc, forming the ZA chancl (rigure 2B). Fhally, in many bromodofew ot including the BET subfamily members and a known as the 'WPF She' is found (Figure 2C). THe ( achieve selectivity between BET and non-BET bromodomains [32].
Bromodomain-containing proteins (BCPs) are often deregulated in disease, and their bromodomains appear to have crucial roles to disease mechanism. Among the classes of $\mathrm{BCPs}$ that have been linked to disease are transcriptor co-regulators (e.g., BET protein BRD4 and ATAD2), transcriptional repressors (e.g., BAZ2A also known as TIP5), chromatin-remodeling factors (e.g., BAZIA, BPTF, CECR2), histone acetyltranserases (e.g., CREBBP and EP300) and E3 ubiquitin gases (e.g., TRIM24) among others. Association of the developrent of bromodomain inhibitors for drug discovers purposes [23]. Over the past 5 years, many inhibithe for ented. The BET subfamily, which comprises BRDT), hums (BRD2, BRD3, BRD4 and la ble to specificlly recognize different actyd terns in $\mathrm{H} 3$ and $\mathrm{H} 4$ histone tails 333 . . (t) be distinct. BET proteins are also likely to bue distinct physiol forming differen prote protein interactions (PPIs) and controlling specific regulatory networks [3]. For example, BRD4 acts as a transcription co-regulator [35.36]: BRD3 binds to acetyated GATA1 and regulates erythroid target genes [37]; while BRD2 is involved in cell-cycle progression $[38$, mong other processes [39].

To date, ten compounds blocking PPIs of BET bromodomains have entered clinical trials. One of them, RVX-208 (Figure 1, also named apabetalone and RVX000222) has reached Phase III clinical trias in autumn 2015 (NCT01728467), being the most advanced one. RVX-208, developed by Resverlogix Corp., has been evaluated in a total of seven clinical trals for the treatment of atherosclerosis and associated cardiovascular disease. In clinical Phase II, RVXA1 increased HDL-cholesterol and apolipoprotei A1 levels, as well as decreased the incidence of major

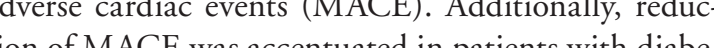
The ors have also reached Phase II clinical trials (OTX015, BMS-9861158 and CSK525762). OTX015 (Figure, eveloped by OncoEthix and Merck is innolved in for different clinical mia and he dranced solid tumors (NCT02259114), 12582), liobastoma multiforme (NCT02296476) and in conbination with azacitidine in patients and in comacute myeloid leukemia that are not candidates for standar inte ling inction (NCT02303782). 


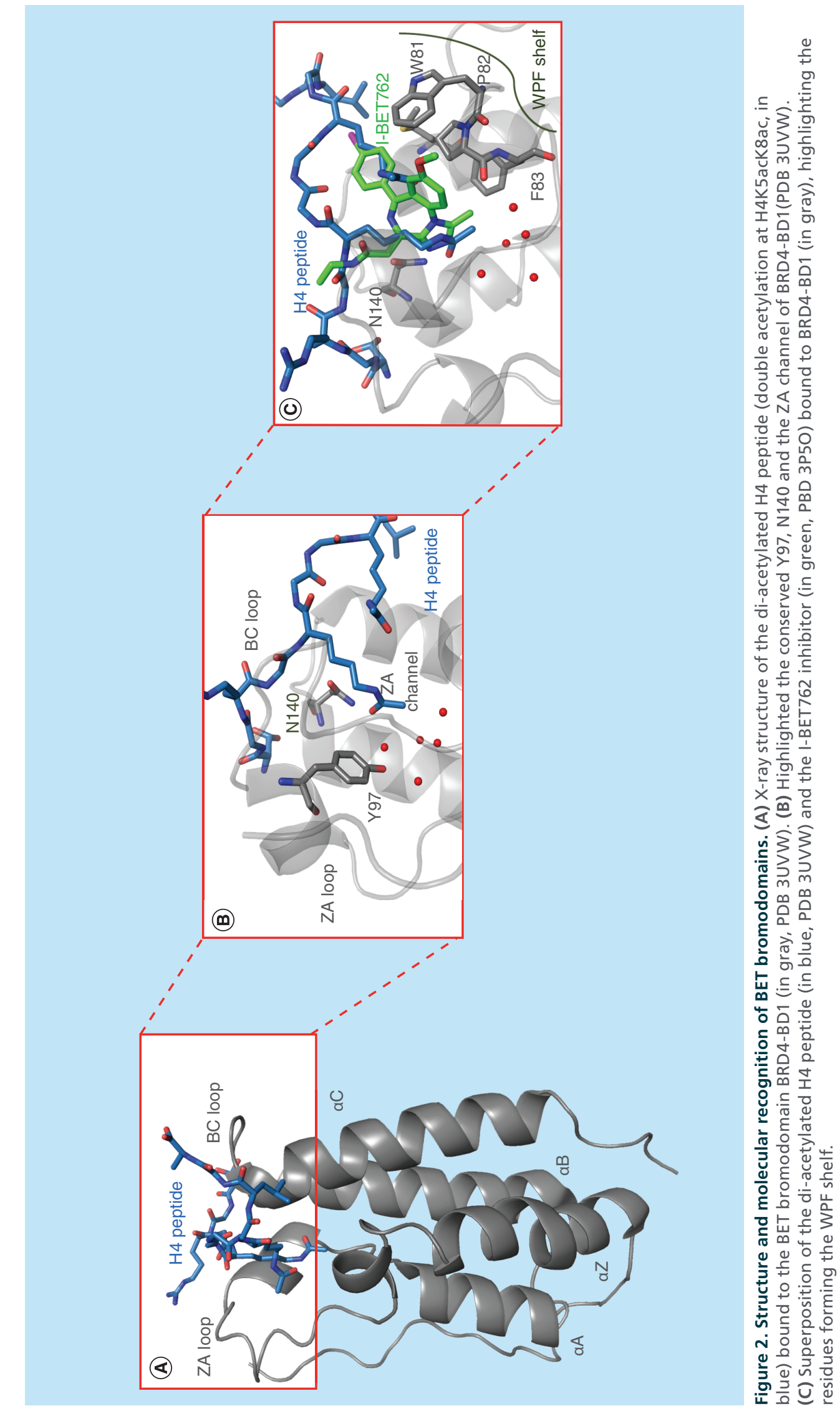

BMS-986158 (structure undisclosed) has been tested have been described with compounds able to bind to for multiple cancer indications alone and together bromodomains in the nanomolar range, achievement with paclitaxel (NCT02419417). Finally, GSK525762, of exquisitely selective molecules for each member of also known as I-BET762 (Figure 1) [18] is involved in the family has proven arduous. BET bromodomains two clinical trials: one to investigate safety, pharma- are representative examples of this problem. Although cokinetics, pharmacodynamics and clinical activ- dozens of potent compounds have been developed in ity in patients with NUT midline carcinoma and the last few years, these are broadly pan-BET selective other cancers (NCT01587703) and a second one for and none of them is able to bind in a selective way to a patients with solid tumors with hematologic malignan- single member of the BET subfamily. In this context, cies (NCT01943851). In addition to these four mol- several compounds with a poly-pharmacology profile ecules, six other BET inhibitors have recently entered for several bromodomains have been described, includPhase I clinical trials and are being studied for both solid ing a compound so-called bromosporine which showed tumors and henatological malignancies: two com- reasonable binding a affinity for different bromodomain pounds 010 [32] (NCT02308761, NCT01987362) and CPI- inhibitors with similar pharmacology but orthogonal 0610 [40] (Figure 1, NCT01949883, NCT02157636, chemotypes lends confidence to the interpretation of NCT02158858); GS-5829 (structure undisclosed NC10239261, NCT02607228); BAY1238097 (struc( (sicher

Despite achieving several compounds in clinical minimize insurgency of drug resistance [21,45]. Guide

drugs, safety is paramount and a level of promiscuity may even be beneficial to their efficacy profile and to entials, the devis major challenge due to hack of single target selectiv- the taret family. For example, Cohen has described ity Chemical probes are small molecules that elicit a specific desiable criteria for kinase probes wor. The cellular response by interacting with a specific protein Structural Genomics Consortium (SGC) has proinside the cell. In doing so, chemical probes can be posed the following criteria for a drug-like compound used to explore the role of that particular protein in to be nominated as a chemical probe: IC or $K_{4}<100$ biological systems [41]. High-quality chemical probes nM, selectivity $>30$-fold over proteins in the same must fulfill certain requirements that drugs do not family and significant cellular activity below $1 \mu \mathrm{M}$ necessarily do. Drugs must be safe and effective at matching quite well with the guidelines proposed by treating disease, in contrast chemical probes must Workman et al. for high-quality selective chemical have a defined mechanism of action and should be as probes [47]. For other subfamilies outside of BET broselective as possible, ideally for a single target [21.42]. modomains, GlaxoSmithKline (GSK) has proposed The recent Chemical Probes Portal 431 provides infor- that a minimum selectivity window of 2 and prefermation and guidelines on the criteria for establish- ably 3 logs over BET bromodomains is required to ing such high-quality chemical probes [21]. The web ensure that an inhibitor has no cross-reactivity and portal alcady contains several bromodomain inhibi- that any biological response is not confounded by ors and could be an invaluable tool for the scientific the strong BET-dependent response expected due to any BET off-target activity [48]. Large-scale in vitro The problem of chemical probe on-target $\quad \begin{aligned} & \text { selectivity profiling against other targets is also rec- } \\ & \text { ommended, for example, against panels of kinases and }\end{aligned}$ selectivity in bromodomains GPCRs [49].

The high similarity within the different subfamilies of The typical strategy to gain selectivity in bromodobromodomains and in particular at the level of their mains has been to start drug discovery campaigns from KAc binding sites poses inherent challenges for the fagm hens or anchoing scaffolds that bind to the KAc development of bo

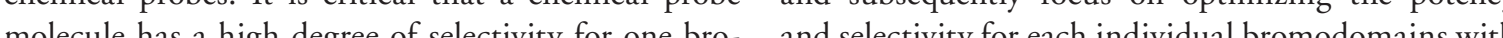

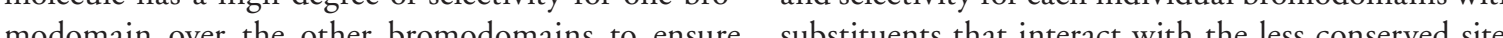
that biogical inights can be confidently ascribed to at the edges of the KAc binding site. This strategy the function of the particular bromodomain of inter- has been successful aginst several bromodomains a est. Although for some subfamilies very high affinities described next. 


\section{Selectivity for BETs versus non-BET}

bromodomains

Selectivity between different subfamilies of bromodomains has been achieved so far with a number of inhibiing sites, and by axploiting strerunces in the KAc bindspecific for certain subfamilies. In fact, Vidler $e t a l$. [51] proposed a classification of human bromodomains based on the structural features that vary across the KAc binding sites, to complement that derived from new classification, ef the entire domain [24]. In this per classification, eight clusters were defined by the presence of a tnique signature of up to three amino peid residues in the binding site, shared by all memful whean group. This classification should prove useperen whe potential to identify possibilities to transfer hit matter from one bromodomain to another. Although selectiv-
ity within members of a given group is also desired, this section will focus on selectivity across different groups with special emphasis on selectivity between BET Wrem

\section{BET bromodomains}

Since the pionering publication of the two triazolodiazepine based BET-selective inhibitors [17187, various inhibitors of this subfamily have been developed, as extennively reviewed elsewhere [23.32 3950505253]. BET bromodomains represent a highly druggable subfamily characterized by a long and accessible ZA channel and by the abovementioned WPF shelf (Figure 2B \& C). All BET bromodomains share this conserved motif, and filling the region of space adjacent to the WPF shelf with a small molecule has proven critical to achieve high binding affinities and to gain selectivity over non-BET bromodomains [54]. In general, BET inhibitors show a very high selectivity profile over members of other subfamilies. A few compounds have, however, exhibited off-target binding affinity for the CREBBP bromodomain comparable to that for BET bromodomains, for example, PFI(Supplementary Figure 1) [55], I-BET726 (also named GSK1324726A) (Supplementary Figure 1) [56], XD14 (Supplementary Figure 1) [57,58], the 3,5-dimethylisoxazole derivatives (Supplementary Figure 1) [59,60] and a recent
$\gamma$-carboline-based chemical series described by Ran et al. $\gamma$-carboline-based chemical series described by Ran $e t a l$.
(Supplementary Figure 1) [6]. Additionally, a novel series of (Supplementary Figure 1) [6]. Additionally, a novel series of compounds described by McKeown et al. demonstrated similar potency for BRD4-BD1 than for the first bromodomain of TAF1 [62].

CREBBP/EP300 bromodomains

Achieving selectivity between CREBBP/EP300 and bromodomains all possess long ZA loops bearing simiar residues facing the KAc binding site. However a few residues differ between the CREBBP and the BET bromodomains binding sites [60]. First, W81 from the top of the WPF shelf of BET bromodomains corresponds to a Leu in the CREBBP, making this part of the binding site of CREBBP more suitable in principle accommodate bulkier ligands. Another residue that Considered to be key for attaining selectivity toward CREBBP is R1173, located at the entrance of the Cinding site of this bromodomain. This residue coresponds to D145 in BRD4-BDI. Some selectivity fo 作 to Several CREBBP inhibitors have been described in recent years, however, in most cases it has proven difficult to achieve strong selectivity over BET bromodoCREBBP was desiber by CRebB was described by Rooney et al. [63]. The best Titration reported, $(R)-2\left(K_{d}=390 \mathrm{nM}\right.$ by lsothermal electivity over a few selocted bro $3 B)$ show good che

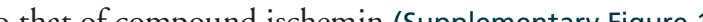
developed previously [6] In both cases, x-ray cyystal strucres (PDB 4 NYW for $(R)-2$ and 2L 84 for ischen. evealed the aility of these compounds to form interactons with the guanidinium group of $\mathrm{R} 1173$ in the $\mathrm{BC}$ oop of CREBBP (Figure 3B).

Hay et al. described SGC-CBP30 (Figure 3B), highly potent $\left(K_{\mathrm{d}}=21 \mathrm{nM}\right.$ by ITC) 3,5-dimethylisoxazole-based inhibitor for CREBBP bromodomain. SGCBP30 displays 40-fold selectivity over BRD4-BD CBd high selectivity over the other BET bromodomain 的 (Pay structure of SGC-CBP30 complexes to CREBBP (PDB 4NR7) highlighted the presence of a cation- $\pi$ (ntidino group of R1173 and the inhibitor, after a structural reor(the inhibitor (Figure 3B). More the same group disclosed an X-ray structure withe the benzoaxazepine-based inhibitor I-CBP112 (

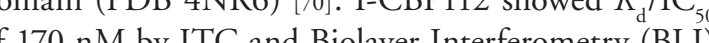
( f $625 \mathrm{nM}$ for he EP300 brom

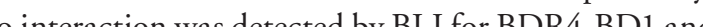
BRD2-BD2. Agan the ayl goup of I-CBP112 inercts with R173 (Figure 3B). For these two las series f elective compounds, the ability to engage in strong terion in the inhibitor aryl ring proved crucial [71].
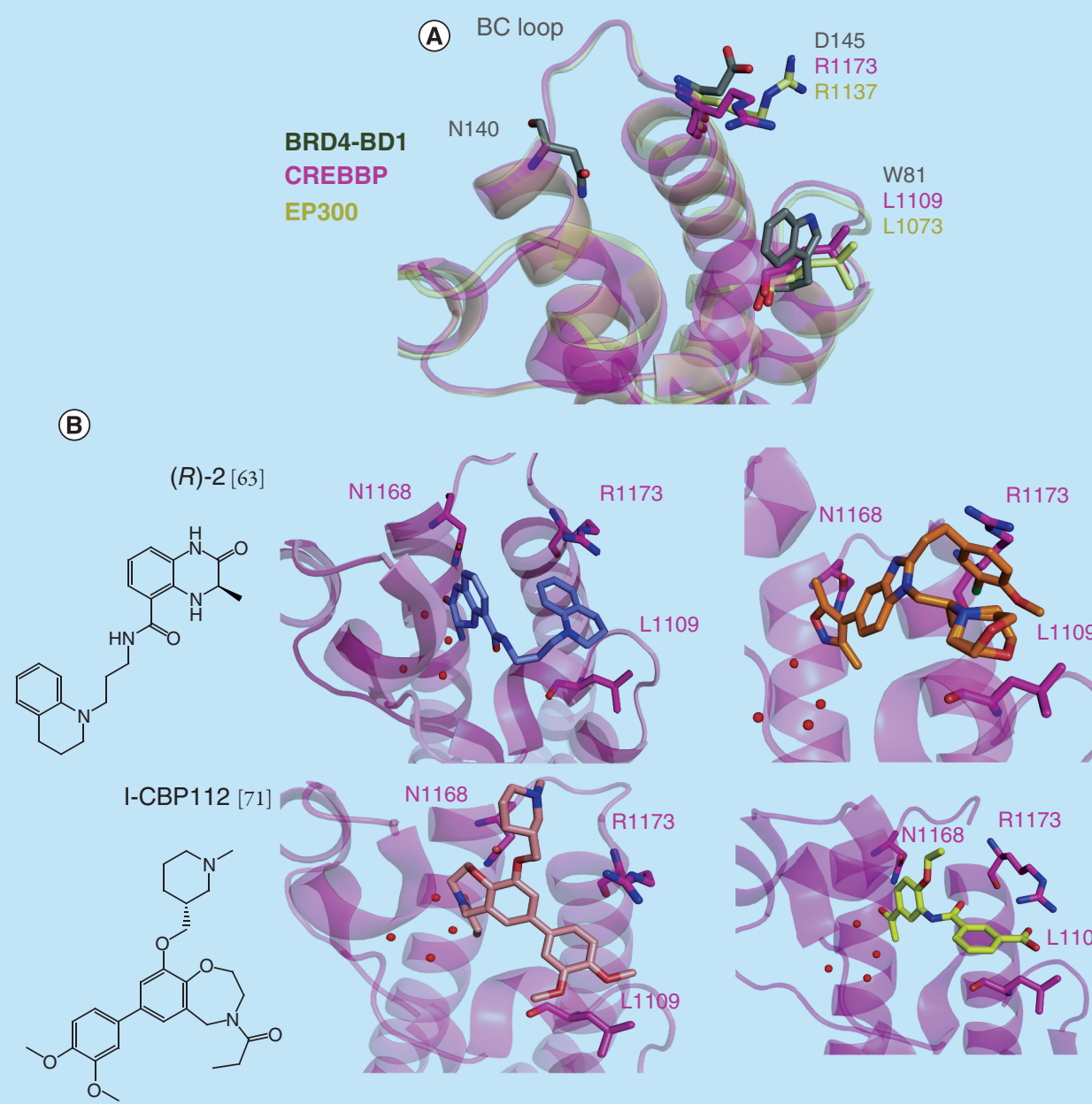

SGC-CBP30 [64]

Figure 3. CREBBP/EP300 bromodomain. (A) Superposition of the $x$-ray structure of CREBBP (in pink, PDB 4NYW), BRD4-BD1 (in gray, PDB 3 UVW) and EP300 (in yellow, PDB 5BT3). (B) X-ray structure of the ligands (R)-2 (PDB 4NYW), SGC-CBP30 (PDB 4NR7), I-CBP112 in the PDB 4NR7.

The importance of R1173 as a key residue to gain between CREBBP and EP300 bromodomains is ye selectivity for CREBBP against BET bromodo- to be described, and could prove far-fetched given the mains was reinforced by a new series of ligands dis- very high sequence identify and binding site similarity covered by an in silico approach, with $K_{\mathrm{d}} \mathrm{s}$ down to between these two domains (Figure 3A). nanomolar range for CREBBP bromodomain $[72,73]$.
Selectivity factors of $>65,59$ and 48 (compounds BRD7 \& BRD9 bromodomains 6,19 and 21, respectively in the original paper, The elusive biological functions of BRD7 and BRD9 Figure $3 B$ \& Supplementary Figure 1) were determined have motivated the development of selective chemical as BRD4-BD1- $K_{\mathrm{d}} / C R E B B P-K_{\mathrm{d}}$ ratio by competition probes against the bromodomains of these proteins. binding [72]. Finite-difference Poisson calculations The crystal structure of BRD 9 revealed a differen on the min the orginal pap (72), also nat of showed that half of the total electrostatic interac- hydrophobic cavity. Additionally the replacement of

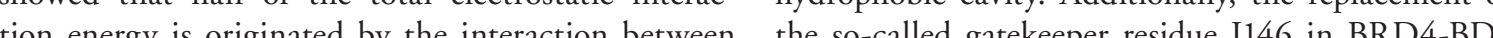
the carboxylic aid of the compound and the R1173 with Y222 in BRD9 results in blocking the access of CREBBP (Figure 3B), Although high selectivity the 'CFF' hydrophobic region that corresponds to the 
important structural differences that were deemed rimetry (DSF) against all expressible bromodomains exploitable for ligand design (Figure 4A) [74l. Several BRD9 inhibitors have been reported. To bilization with other bromodomains [75]. Additionally, date four distinct chemical series of compounds have LP99 was found to be inactive versus 55 receptors and been described that show selectivity toward BRD7 and ion channels (CEREP panel) at $10 \mu \mathrm{M}$. The co-crystal BRD9 [75-79]. Starting from a verolactam fragment, structure of LP99 with BRD9 (PDB 4Z6I) confirmed Clark et al. aimed to extend interactions to the different that LP99 is stabilized by hydrophobic and aromatic hydrophobic regions between F160 and 1169 of BRD9. residues in the KAc binding pocket and elucidated This approach ultimately yielded LP99 (Figure 4B), a the structural basis for the stereospecific activity of potent and selective inhibitor of BRD7 and BRD9, (2R,3S)-LP99 (Figure 4B).

with a $K_{\mathrm{d}}$ against BRD9 and BRD7 of 99 and 909 Another series of compounds that have shown selecfiled for selectivity by Differential Scanning Fluo- the BAZ2A/BAZ2B inhibitor GSK2801 (discussed

(A)

(B)

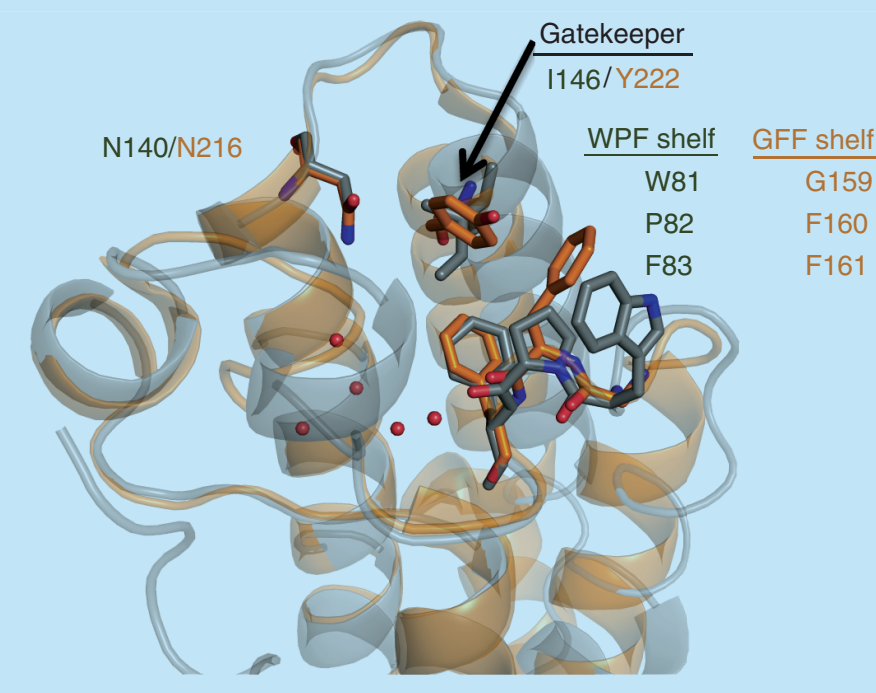

LP99 775
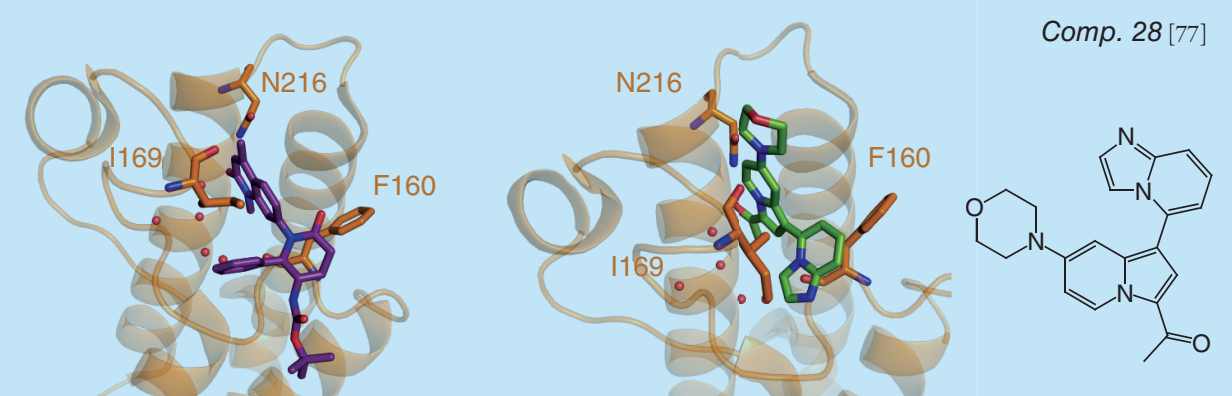

I-BRD9 [76]
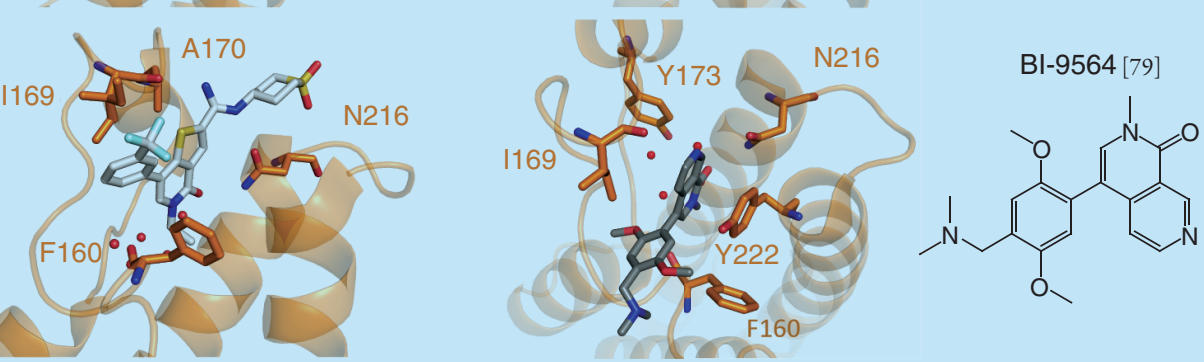

figure 4. BRD9 bromodomain. (A) Superposition of the x-ray structure of BRD9 (in orange, PDB 4Z61) and BRD4-BD1 (in gray, PDB

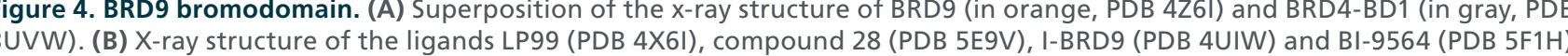
bound to BRD9 (in orange). ater) $[77,78$. . The authors noted that the indolizine fragment of GSK2801 had also affinity for BRD9. After optimization of the starting fragment, compound 28 (from the original paper [77], Figure 4B) was found to be highly potent against $\mathrm{BRD} 9\left(K_{\mathrm{d}}=68 \mathrm{nM}\right)$ and slightly
less potent for $\mathrm{BRD} 7\left(K_{\mathrm{d}}=368 \mathrm{nM}\right)$. The broade selectivity of this compound was assessed by DSF. Compound 28 is selective over most of the other bromodomain subfamilies, with modest affinity only for BRPF1B, CREBBP/p300 and FALZ. After determination of the x-ray structure of compound 28 bound to BRD9 (PDB 5E9V, Figure 4B), the selectivity observed could be rationalized by an interaction formed by the C-I imidazopyridine moiety with the hydrophobic region sandwiched between I169 and F160 which is much narrower in the BET bromodomain (Figure 4B) [77].

The high similarity between $\mathrm{BRD} 7$ and $\mathrm{BRD} 9$ bromodomains (around 80\% sequence homology; 36\% in terms of overall residues) $[7,7]$ makes the develop domains a difficult task. However, this was achieved recently with the compound I-BRD9 (Figure 4B) [76]. LBRD9 was in design, leading to greater than 700 -fold selectivity over the BET subfamily members and 200-fold over the highly homologous BRD7 ( $K$ [BRD9, DiscoveRx] $1.9 \mathrm{nM}, K[\mathrm{BRD} 7$, DiscoveRx] $=380 \mathrm{nM}, K[\mathrm{BRD} 4$ BD1, DiscoveRx] = $1400 \mathrm{nM}$ ) [76797]. Several $\mathrm{x}$-ray structures bound to BRD9 and BRD4-BD1 from several compounds during the extensive SAR process were solved in order to gain insights into the structura features that are responsible for the observed selectivity. Considering the basic nature of the amidine moiety of I-BRD9 (Figure 4B), it was proposed that whe charged it would sit more favorably in the less hydrophobic environment alongside Al70 of BRD9 than beside L94 of BRD4-BDI (Figure 4B, PDB 4UIW). The additional selectivity of I-BRD9 for BRD9 ove $\mathrm{BRD} 7$ is, however, difficult to rationalize since only an NMR structure of BRD7 (PDB 217K) is reported to date [80]. Potential contributions to the observed selec tivity arise from differences in the constituent amin acids and in the architecture of the GFF region of BRD) (G15) in BRD corresponds to A154 in BRD7) and the ZA) loop (A162 in BRD corresponds to S157

Ver Veloped using fragment-based optimizatio with SGC-Oxford and Cold Spring Harbor Laboratory (BI-7273 and BL-9564) [791. The x-ray structure of BL-9564 bound to BRD9 (PDB 5F1 H, hows that the ligand makes water-bridged interactions with the specific BRD9 gatekeeper residue Y222, while keeping a double hydrogen bond with N216 and a water-mediated hydrogen bond with Y173. The compounds are potent $\left(K_{\mathrm{d}}\right.$ in the $10^{-9}-10^{-8} \mathrm{M}$ range), highly of kinase (>1000-fold) against BRD4-BD1 and a pane and PK profiles, block AML cancer cell proliferatio at $\sim 1 \mu \mathrm{M}$ and display efficacious antitumor activity In a xenograft model of human AML [79], providing the most high-quality chemical probes for BRD7/9 Orsted to date.

Other in lso been reported. These compounds either show 作 ay subfamily [81], which se the unsuitable as chemical probes. Interest(s) $f$ modest selectivity, were able to induce an unot dented rearrangement in the KAc recogition XY8), which was no f the same compound bound to BRD4-BD1 (PDB XY9). S rotated $120^{\circ}$ thus blocking the $\mathrm{ZA}$-channel of protein resulting in a steric clash around the ligands. These conformational changes could be exploied in principle to achieve selectivity for BRD7/9.

BAZ bromodomains

The BAZ subfamily of bromodomains (BAZ2A and BAZ2B) [82] had been considered one of the least druggable in the human genome [51]. They are characterized by a shorter ZA loop, making the pocket fairly open and reducing its druggability, and by lack of the ZA channel. On the other hand, the residue correspond ing to M149 of BRD4-BD1 is much smaller in BAZ2B (A) of the key Trp in the WPF shelf,

In 2013, the fist sures In 2013, the first series of fragments with hig In were reported, which led to the structure-guided (PDit

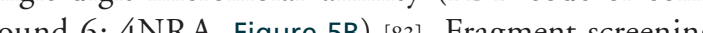
6: of the first nanol (Figure 5B), against the protein [781. An indolizine frag( ecognition interaction was grown and opimied to GSK2801 based on structural information A cocrystal structure (PDB 4RVR) showed that W1887 in BAZ2B is able to accommodate favorable $\pi$-stacking 
(A)

BRD4-BD1

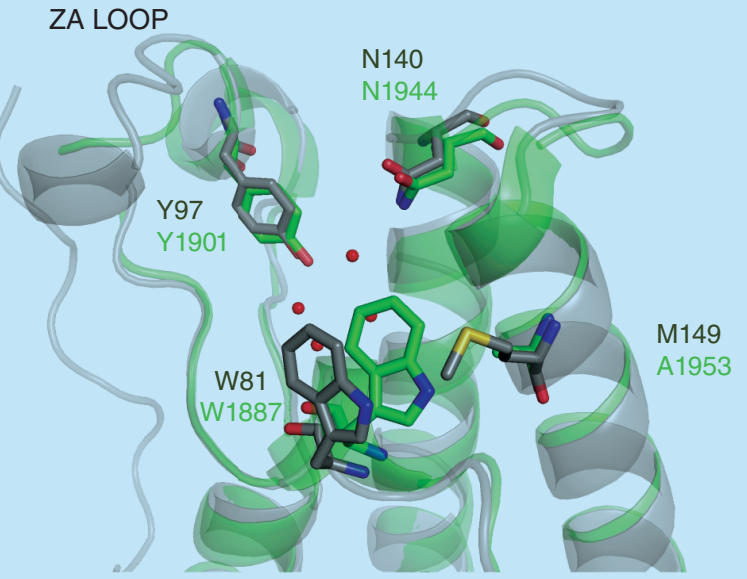

(B)

Comp. $6[83]$

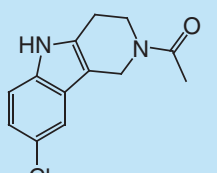
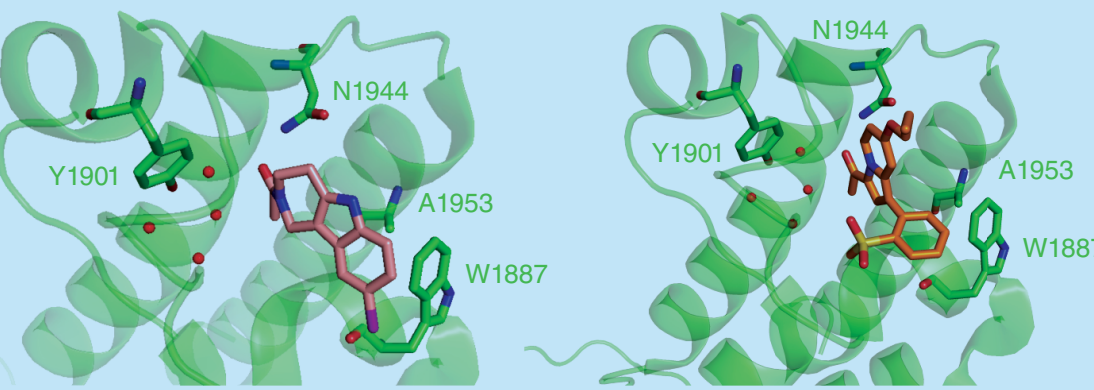

$$
\text { (178] }
$$

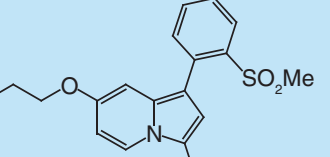

$=0$
BAZ2-ICR [84]

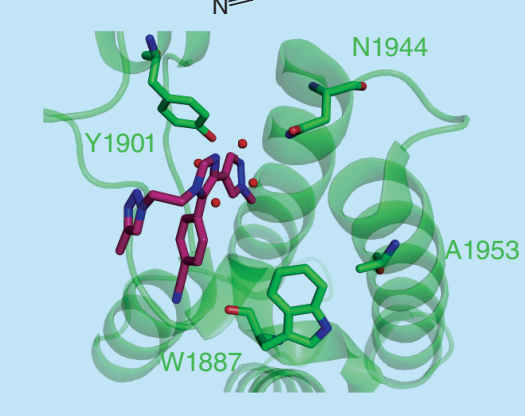

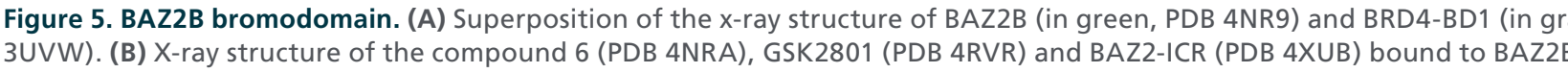

interactions with the inhibitor (Figure 5B). Bulky sub- Noteworthy is the intramolecular $\pi$-stacking interacstituents in this part of the ligand introduced steric tions formed by the compound in its bound conforclashes against BET bromodomains, increasing selec- mation. This structural feature, often referred to as tivity as a result. GSK2801 showed $K_{\mathrm{d}} \mathrm{s}$ of 140 and 'hydrophobic collapse', could help to achieve potency $260 \mathrm{nM}$ by ITC against BAZ2B and BAZ2A bro- against other less druggable bromodomains, since it modomains, respectively. Only BRD9 and TAF1(L) can provide shape complementary and extensive conbromodomains were detected as the major off-targets, tacts in the case of more open and solvent-exposed after assessment of the GSK2801 selectivity for all binding sites, featuring less enclosed pockets, as is the human bromodomains by two orthogonal biophysical case with BAZ2B.

techniques, DSF and BLI.

In a parallel effort, Drouin et al. described BAZ2- BRPF bromodomains

ICR (Figure 5B), an inhibitor that targets BAZ2A and The BRPF bromodomains KAc site closely resemble $B A Z 2 B$ in the nanomolar range achieving highly selec- the BET and CREBBP bromodomains. This structural CERC2,

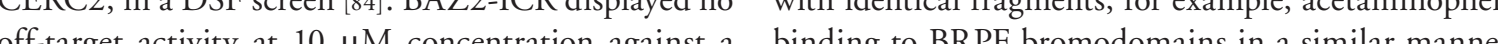

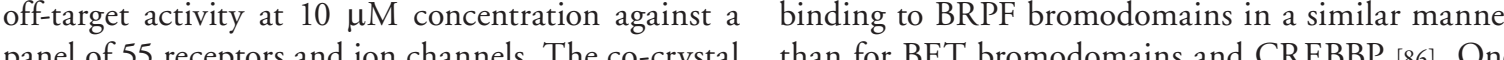
structure (PDB 4XUB) provided one of the firsexam- signifant difference between BRD4 and BRPF1 is ples of a pyrazole moiety acting a a KAc mimetic eff- the hydrophobic 'patekeeper' residue that foms on ciently flling out
V439 in BRD4-BD2 (Table 1). In contrast, a larger Phe This replacement promotes the selectivity seen in the The best in class of this series, compound 3 (in the original paper, Figure 7B) [48], showed a $p \mathrm{IC}_{50}$ for BRPF1 of for BRPF1 over BRPF2 and BRPF3 $\left(p \mathrm{C}_{50}\right.$ of 5.1 and $<4$, respectively) could be achieved with compound 3 , which was unexpected because the BRPF bromodomains themselves are highly conserved and share $>65 \%$ sequence identity. This observation could be rationalized by the substitution of S592 in BRPF2 or N619 in BRPF3 with P658 in BRPF1. Compound 3 was tested in the BROMOscan pand of 35 bromodomains and howed high selectivity over other bromodomains [48] Keeping the 1,3-dimethyl benzimidazolone core,
scientists at the SGC reported OF-1 and PFI-4 (Figure 6). OF-1 (PDB 5FG4), developed in collaboration with Pfizer, showed good affinity to all of the ration with $\mathrm{Phec}$, showed good afficy to all of the the bromodons closest off torget effects wore found agins BRD4 (39-fold selectivity) and TIF1a (50\% inhibition at $20 \mu \mathrm{M}$ in an alphascreen assay). PFI-4 (PDB 5FG5, Figure 6) binds to BRPF1B with a $K_{\mathrm{d}}$ of $13 \mathrm{nM}$ by ITC [87]. Selectivity data have not been published yet for PFI-4. residue occupies this position in the BRPF subfamily. 1,3-dimethyl benzimidazolones-based series described by Demont et al. (Figure 6 \& Figure 7A, PDB 4UYE). 7.1, while the $p \mathrm{IC}_{50}$ for BRD4 was 4.3 [48]. Selectivity

With the initial aim to obtain selective TRIM24 inhibitors, two molecules have been developed recently that are selective for BRPF and TRIM24, named ACCS-9571 and compound 34 (Figure 6), respectively $[88,89]$. Selectivity over BRD4 was ensured by the structural similarity of the starting fragments to the ones used to develop BRPF inhibitors [48], however, his meant that no selectivity for TRIM24 over BRPF tromodomains could be obtained. A trend was seen both cases, in that introduction of bulky group at the 6-para-methoxy position of the 1,3-dimethy benzimidazolone core scaffold increased dramatically the anting towning

Only one nonrelated 1,3-dimethyl benzimidazolon compound has been disclosed that is able to bind in a selective manner the BRPF bromodomains. NI-57 (Figure 6) [87] binds to the all BRPF subfamily mem

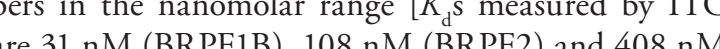
(BRPF2)] N -57 ), $108 \mathrm{nM}$ (BRT2) and $408 \mathrm{nM}$ (B. tiophysica est off-target offect of $\mathrm{NL}-57$ is against BRD9 (32-fold

ATAD2 bromodomain

Similar to BAZ2B, ATAD2 has been considered a poorly druggable bromodomain since the KAc binding

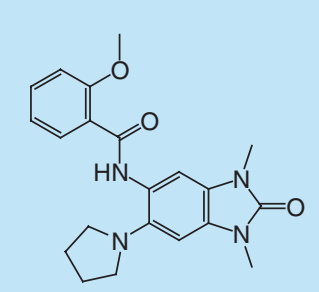

OF-1

PFI-4
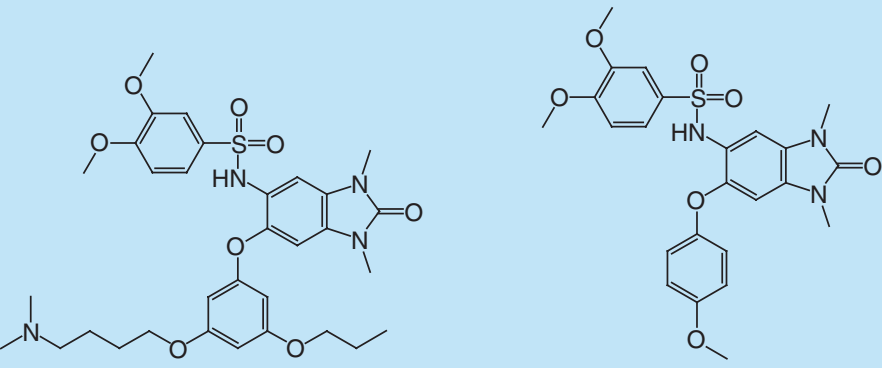

IACS-9571 $1^{180}$

Comp. 34 $34^{189}$

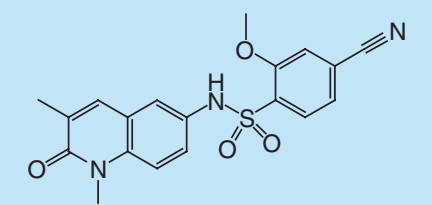

$\mathrm{NI}-57$

Figure 6. BRPF inhibitors. Chemical structure representation of OF-1, PFI-4, IACS-9571, compound 34 and NI-57. 

site is significantly divergent with respect to those of $>100$-fold selectivity over the BET bromodomains. che responsible for KAc recognition, the KAc binding site (PDB code of compound 42: 5A83, Figure 7B). Howis more open, polar and flexible than for example the ever, the high potency and selectivity of compound 38 one of BET bromodomains. When compared with came at the expense of greater hydrophilicity that was BRD4-BD1, only three of seven residues lining the solved in part with compound $46\left(\mathrm{pIC}_{50} \mathrm{ATAD} 2=6.5\right.$ KAc binding pocket are shared, and the ZA loop is by TR-FRET, Supplementary Figure 1). Selectivity also two residues shorter than in BRD4. Additionally, of compound 46 over the BET bromodomains was W81 from the WPF is replaced by R1007, while M149 confirmed by Bromoscan with a window of at least is replaced by R1077. In ATAD2, the region corre- >400-fold ( $p$ Ki by BROMOscan 7.7 against ATAD2 sponding to the WPF shelf has been named 'RVF shelf' and 5.1 against BRD4-BD1). For the other bromodo(R1007-V1008-F1009) (Figure 78). Several screening mains tested, the highest activity was with the second campaigns have been performed to find fragment- bromodomains of TAFI and TAFIL ( $p$ Ki 7.3 and 6.9 size hits for ATAD2 990,91 , which yielded a number of respectively, by BROMOscan). In addition, chemoproweak-binding compounds. In parallel efforts, after a teomics pulldown experiments were performed and $K_{\mathrm{d}}$ ong and careful structure-based optimization a group for the off-target bromodomains were determined [92]. at GSK developed potent and selective naphthyridoneased ATAD2 inhibitors [92.93]. In a first step, the Other bromodomains

athors were able to find low micromolar inhibitors for Few other bromodomains have been selectively tar-

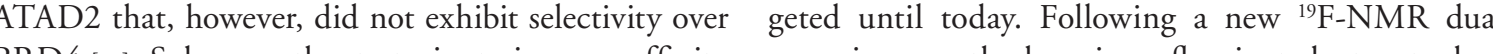

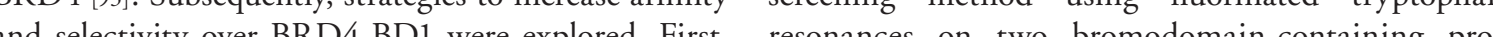
complementaity with the RVF shelf was optimized teins Urick ot al discovered the first selective BPTF with appropiate polar substitutions in the compounds. inhibitor called AU1 (Supplementary Figure 1), from a Second additional interactions with the backbone NH library of 229 selected small moleculesscener aga group of D1014 in the ZA loop were introduced, yield- BRD4-BD1 and BPTF [94]. SGC and Pfizer have coling the best compound of this series, compound 38 (in laborated to develop PFI-3 (Supplementary Figure 1, the original paper, Supplementary Figure 1) [92], with PDB 5DKC [SMARCA2] and 5DKD [SMARCA4]), double-digit nanomolar binding affinities for ATAD2 a selective chemical probe for SMARCA 2/4 (89 nM ( pIC $_{50} \mathrm{ATAD} 2=6.9$ by TR-FRET) and showing for SMARCA4 by ITC) and PB1(5) (48 nM by ITC)

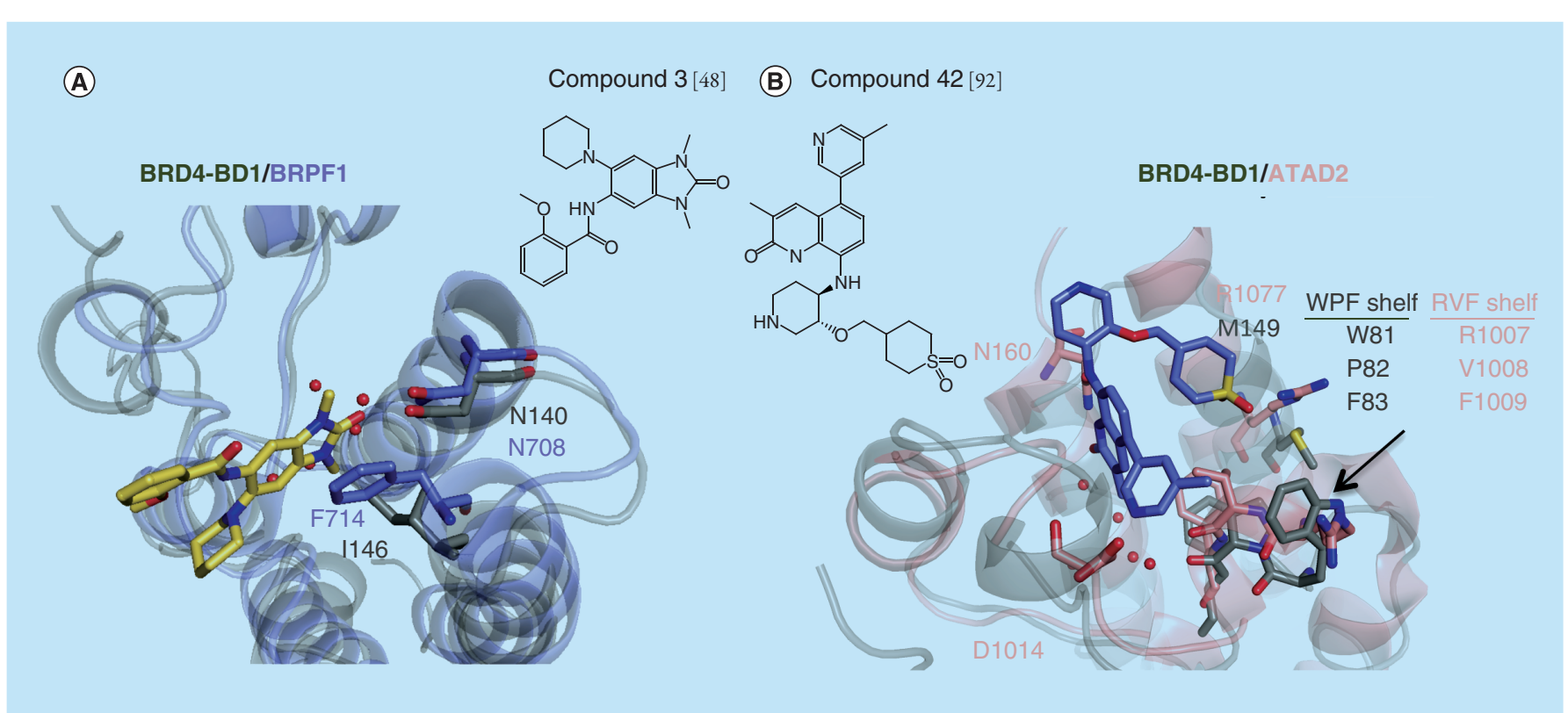

Figure 7. BRPF and ATAD2 bromodomains. (A) Superposition of the $x$-ray structures of compound 3 (in yellow) bound to BRPF1 (in purple, PDB 4UYE) and BRD4-BD1 (in gray, PDB 3UVW). (B) Superposition of the x-ray structures of compound 42 (in purple) bound to purple, PDB 4UYE) and BRD4-BD1 (in gray, PDB 3UVW). (B) Superpos
ATAD2 (in light pink, PDB 5A83) and BRD4-BD1 (in gray, PDB 3UVW). bromodomains [95]. No interaction was observed with other bromodomains by DSF, and there was no crossreactivity in a kinase panel of 36 kinases [95]. Despite the achieved potency and pan-selectivity, target bro-
modomain inhibition by PFI-3 did not impart the expected antiproliferative phenotype in relevant cancer cell lines, in contrast to what was observed with target knockdown by RNAi [95]. In another collaborative projinhibitor NVS-CERC2-1 (Supplementary Figure 1) [87]. With an affinity of two-digit nanomolar and no interaction with the rest of the bromodomains in a BRD panel (48 targets), NVS-CERC2-1 is the only example to date of a chemical probe for this subfamily of bromodomain

Selectivity within BET bromodomains: BD1

All BET proteins are characterized by two $\mathrm{N}$-terminal tandem domains (BD1 and BD2) followed by an extra terminal domain (ET domain). While BDIs and BD2s mepane shan identity is observed, sugresting distinct evolion identity is obsersed, suggesting distinct evolutionary BET bromodonins exhibit 95\% sequence idenity at the KAc binding pocket [97]. Sequence compar son between the two bromodomains of BRD 4 in the proximity of the $K A_{c}$ peptide-binding site (Figure $8 A$ Table 1) shows three crucial residue positions that differ

- $\mathrm{Q} 85$ in BD1 is a Lys residue in BD2 (K378);

D144 in the BC loop of BD1 is a His residue in $\mathrm{BD} 2$ (H437); and

- I146 is a Val in BD2 (V439).

Although BET BD1 and BD2 show a high sequence similarity in the substrate-binding site, they exhibit different patterns of recognition of acetylated histone peptide target sequences [24]. A single BD1 or BD2 of
BRD4 is individually able to interact with acetylated H4 peptide, however, only BD1 was found to recognize specifically N-terminal acetylated $\mathrm{H} 4$ peptides in a sequence-dependent manner, while BD2s were found to be more promiscuous [24]. As a general fea-
ture, BD1s of BET proteins appear to have a role in ture, BDIs of BET protens appear to have a role in BD2s could ha bro der speifiry towat acety substrates. The presence of two additionl conser regions one containing the N-terminal cluster of casein kinase II phophas ites (NPS) and one cocos. ing resides-enribed interaction don ated downstream of $\mathrm{BD} 2$ in all BET proteins, would suggest that $\mathrm{BD} 1$ and $\mathrm{BD} 2$ may be differentially egulated by post-translational modification [98]. Several studies have shown that each BET bromodomain has a distinct function in the regulation of ordered gene transcription in chromatin possibly consequent the interaction with lysine-acetylated histones or with other partner proteins. Gamsjaeger et al. showed that the two acetylated lysines in a sequence adjacent to the DNA-binding domain of the hematopoietic transcripthe $\mathrm{BD}$ a does not play she significant role in the recognition [37,99]. Shi et al. sto selectivity of $>$ peptides could bind BRD4-BD2 with stiver polarization assay, suggesting that Twist preferentially interacts with the second bromodomain [100]. The prefsential selectivity of the diacetylated Twist toward D1 was attributed to a charged amino acid residue (D2 4 in BD1 or residues beyond the diacetylation motif [100].

The same structural difference (D144 in BD1 or 4437 in $\mathrm{BD} 2$ ) was highlighted as potentially playing a the observed secectivity of a compound called olinon for BRD4-BD1 over BD2 [97]. Olinone (Figure 8B) is a selecive small-molecule inhibitor of the firs bromodomin of BET proteins, consistently exhibiting over 100 -fold higher binding affinity to $\mathrm{BD} 1(K=3.4 \mu \mathrm{M})$ than $\mathrm{BD} 2$ (no detectable binding) by ITC for all of the BET bromodomains. A tetrahydropyrido indole chemical fragment (MS7972, Supplementary Figure 1) was identified as a hit from an NMR-based screen and showed modest activity as inhibitor of the CREBBP bromodomain [65] Using MS7972 as a starting point, the authors incorporated longer acetamidoalkyl group substituents at the $N$-indole core, mimicking the natural KAc substrate. The $\mathrm{x}$-ray structure of the BRD4-BD1/olinone complex (PDB 4QB3, Figure 8B) revealed that the alkyl group containing four methylene units adopts identical conide side chain of the histone $\mathrm{H} 4 \mathrm{~K} \mathrm{~K} \mathrm{ac} / \mathrm{K} 8 \mathrm{ac}$ peptide whe bound to BRD4-BD1. Crucial for the BD1 selectivity the modified triheterocyclic moiety containing a cyclic amide packs against the side chains of $\mathrm{W} 81$ and $\mathrm{P} 82$ an he opening of the KAc bindine BDI residue DIH4 at he ZA a BC KA I I cor residue in $\mathrm{BD} 2 \mathrm{H} 437$, would be predicted to chs with the cyclic moiety of olinone (Figure 8A).

The ability of olinone to bind pef entilly the firs The to be used as a chemical probe to address how each of 


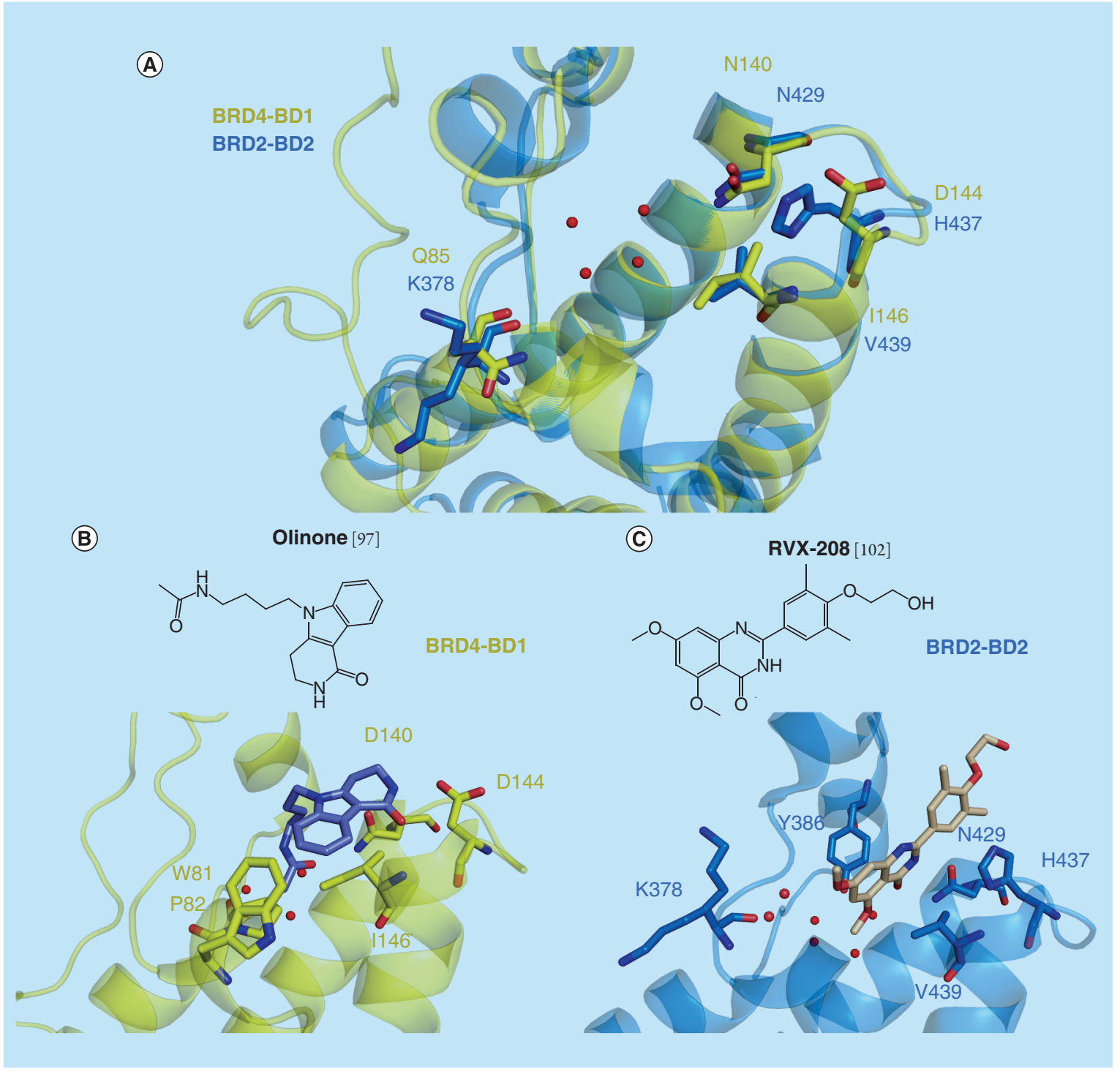

Figure 8. Selectivity between BD1 and BD2 BET bromodomains. (A) Superposition of the $x$-ray structure of BRD4-BD1 (in yellow, PDB 4QB3) and BRD2-BD2 (in blue, PDB 4J1P). (B) $X$-ray structure of olinone bound to the
BRD4-BD1 (in yellow, PDB 4QB3). (C) $x$-ray structure of RVX-208 bound to the BRD2-BD2 (in yellow, PDB 4J1P).

the paired bromodomains of BET proteins may func- BET inhibitors [101]. MS436 (Supplementary Figure 1), tion individually in the control of gene transcription in with an estimated $K \mathrm{i}$ of 30-50 nM for BRD4-BD1, chromatin. In fact, comparison of the effect between and 10-fold selectivity over BD2, was the best inhibito olinone with MS417 (Supplementary Figure 1) that is obtained after extensive lead optimization [101]. MS61 equally potent against $\mathrm{BD1}$ and $\mathrm{BD} 2$ showed dramatic (Supplementary Figure 1) is another diazobenzenedifferences in the biological response of oligodendro- based BET inhibitor with selectivity for BD1 agains cyte progenitor lineage cells to compound treatments: $\mathrm{BD} 2$ more recently described by the same group [97] whereas olinone promoted differentiation, MS417 Surprisingly, MS611 showed 100-fold selectivity only ing and po 2 lly pointing to different . potency of olino and potential off tarets mediating or its biological activity. Preferential binding for BD1 over BD2 has been structiral a malsis is wifferences observed be the basis achieved with another chas of diabobenzenebsed bromedon.
While olinone represents the first selective BD1 inhibitor, RVX-208 (Figure 1 \& Figure 8C) was the first BD2 selective inhibitor reported. RVX-208 (also named RVX000222 and apabetalone) was developed by Resverlogix Corp. and is now in clinical Phase III for the treatment of cardiovascular diseases associated with atherosclerosis and diabetes. RVX-208 is a small molecule that binds to BET bromodomains and competes for acetylated histone $\mathrm{H} 4$ peptide with a hig preference for $\mathrm{BD} 2$ over $\mathrm{BD} 1$. Two papers published around the same time described the biophysical and structural characterization of this small molecule against BET bromodomains [102,103]. Although the results are very consistent along the two papers, some discrepancies about the absolute binding affinities me sured by ITC of RVX-208 against BD1 and BD2 are seen. While Picaud et al. measured 23-fold selectivity
for BRD2 $[K=5790 \mathrm{nM}(\mathrm{BD} 1)$ and $251 \mathrm{nM}(\mathrm{BD} 2)]$ for BRD2 $\left[K_{\mathrm{d}}=5790 \mathrm{nM}(\mathrm{BD} 1)\right.$ and $\left.251 \mathrm{nM}(\mathrm{BD} 2)\right]$ and eightfold selectivity for BRD4 $\left[K_{\mathrm{d}}=1142 \mathrm{nM}\right.$ (BD1) and $135 \mathrm{nM}$ (BD2), McLure et al. measured and $206 \mathrm{nM}(\mathrm{BD} 2)]$ and 30-fold selectivity for BRD4 $\left[K_{\mathrm{d}}=8930 \mathrm{nM}(\mathrm{BD} 1)\right.$ and $\left.303 \mathrm{nM}(\mathrm{BD} 2)\right]$. These differen experiments.

The high-resolution crystal structure of RVX-208 bound to BRD4-BD1 (PDB 4J3I and 4MR4) and BRD2-BD2 (PDB 4JIP and 4MR6) were in a peptide-competitive manner, but unlike pocket it does not occupy the WPF region (Figure $8 C$ ). In all crystal structures, RVX-208 adopts a conserved binding mode. The carbonyl oxygen and the nitrogen atoms of the quinazoline ring system act as the KAc mimetic moiety, forming a hydrogen bond with the conserved N429 (BRD2-BD2) and a water-mediated hydrogen bond with $\mathrm{Y386}$. The BD2 unique residue H433 in BRD2 flips into the KAc binding site packing against the phenyl ring of RVX-208, providing a possible explanation for the tighter affinity for BD2s (Figure 8C). Moreover, RVX-208 makes no direct interactions with residues unique to BD1, except to a water-mediate

Selectivity for the second over the first BET bromodomain using the IBET/(+)-JQ1 triazolodiazepine scaffold was observe by Band at al. in the biology \& chemical genetics appro (ses 'Cheming selectivity' section of this review) [1063 'An an f I-BET762/(+)-JQ1 (compound 28 in al paper [104] Supplementary Figure 1) in which an indole group replaces the $p$-chlorophenyl group of I-BET762/(+)-JQ1 displayed a marked BD2 serce profile (20-fold). The observed selectivity was rationalized by observing in the co-crystal structures two inct conformations (open and closed) for the His side chain (H433 in BRD2-BD2). The newly introduced indole group of the ligand exploits the closed conformation of $\mathrm{H} 433$ by forming an edge-to-face $\pi$-stack that would not be possible with the BD1 Asp residue (PDB 5DFD). These results indicate that BD2 vesus BD1 isoform selectivity could be optimized with the aryl triazolodiazepine scaffold via comprehensive Fin Hnally, several compounds developed by Zenith Epigenetics (structure undisclosed) have also shown BD1. The bectivity for BRD4-BD2 toward BRD460. The best-in-class compound, ZEN297, showed 20 as and potency of BD2-sin (a) hem less attractive drug leads than BET inhiters with $\mathrm{BD} 1$ activity.

\section{Selectivity within the BET subfamily}

The development of an inhibitor selective for one of the bromodomain protein inside the BET subfamily has originated a tremendous interest in the academic and industrial settings. Although dozens of new molecular entities have been published and disclosed in the last 5 years, none of these have exhibted exquisite selectivity for a single BET protein over the others, and little is known about how to achieve this challenging selectivity. This is in part as a result of the high sequence identity within the KAc bindg pocket of BET bromodomains (Table 1). How der, very recently, Raux et al. indicated a differen ynamic behavior of the ZA loop between the differfir xanthine-based inhibitors (Supplementary Figure 1 ) discovered using a 'protein-protein interaction inhbition (2P2I)-oriented' collection of compound was able to yield the first described low micromoar selective inhibitor targeting BRD4-BD1 with greater than tenfold ratio in binding affinity toward ny other BET bromodomain. A potential hydrogen bond between the triazolo fragment of the new that could be nd the BET conserved D88 residue BD1, could in part explain the observed selective profiles. Additiolly, van der Waals inter section with the side chin of the aforementioned Q85 in BRD4- 
the preferential binding observed toward the BD1 Chemical biology \& chemical genetics bromodomain.

The pharmaceutical company Bayer disclosed in The Annual Meeting of The American Association for Cancer Research in 2015 the biochemical characteristics of BAY1238097 [107]. This compound, based on a novel scaffold (structure undisclosed), Previous work on different enzymes, including protein showed $\mathrm{IC}_{50}$ values of $63 \mathrm{nM}$ for BRD4, $609 \mathrm{nM}$ kinases [109,110] provided proof-of-concept for generatfor $\mathrm{BRD} 3$ and $2430 \mathrm{nM}$ for $\mathrm{BRD} 2$, corresponding ing allele-specific cofactors and inhibitors using a soto a selectivity of up to 39-fold for BRD4 versus called 'bump-and-hole' approach. Using site-directed BRD2. Chromatin immunoprecipitation (ChIP) mutagenesis a hole' is introduced in the protein by experiments performed in MOLM-13 (AML) and replacing a natural amino acid with a smaller one, and BAY 3 (MM) cell lines additionally revealed that this is compensated by introducing a bulky hydroBAY1238097 prevented binding of BRD4 to $c$-Myc phobic 'bump' into the natural ligand. This would regulatory region.

Another compound showing a degree of intra-BET duced mutant over the wild-type protein as the latter electivity in vivo is OTX015 (Figure 1), a BET inhibi- should not bind the 'bumped' ligand owing to steric or in clinical Phase III, which binds BET proteins hindrance.

with $\mathrm{IC}_{50}$ around $100 \mathrm{nM}$ [108]. Coude et al. showed Baud et al. successfully developed this approach that OTX015 exposure decreased the expression of to achieve exquisite single target selectivity of BET RRD2 and BRD4 and $c-M Y C$, while BRD3 expres- bromodomain chemical probes [111]. After sequence lecomi centes [nes.

\begin{tabular}{|c|c|c|c|c|}
\hline Motifs & BRD2-BD1 & BRD3-BD1 & BRD4-BD1 & BRD4-BD2 \\
\hline Conserved Asn & N156 & N116 & N140 & N433 \\
\hline Conserved Tyr & Y113 & Y73 & Y97 & Y432 \\
\hline WPF motif & W97 & W57 & W81 & W374 \\
\hline WPF motif & P98 & P58 & P82 & P375 \\
\hline WPF motif & F99 & F59 & F83 & F376 \\
\hline ZA loop & D104 & D64 & D88 & D381 \\
\hline ZA loop & A105 & A65 & A89 & V $382^{+}$ \\
\hline ZA loop & V106 ${ }^{+}$ & $166 t$ & $\mathrm{~V} 90^{+}$ & $E 383^{+}$ \\
\hline ZA loop & K107 & K67 & K91 & A384t \\
\hline ZA loop & L108 & L68 & L92 & L385 \\
\hline ZA loop & G109+ & $\mathrm{N}^{+9^{+}}$ & $\mathrm{N}^{+}{ }^{+}$ & G386 $6^{+}$ \\
\hline ZA loop & L110 & L70 & L94 & L387 \\
\hline ZA channel & $\mathrm{R} 100^{+}$ & Y60 & Q84 $4^{+}$ & Y377 \\
\hline ZA channel & Q101 & Q61 & Q85 & K378 ${ }^{+}$ \\
\hline ZA channel & P102 & P62 & P86 & P379 \\
\hline ZA channel & V103 & V63 & V87 & V380 \\
\hline Gatekeeper & 1162 & 1122 & 1146 & V439' \\
\hline BC loop & T159 & T119 & G143+ & D436 ${ }^{+}$ \\
\hline BC loop & D160 & D120 & D144 & $\mathrm{H}_{437^{+}}$ \\
\hline BC loop & D161 & D121 & D145 & E438 \\
\hline
\end{tabular}

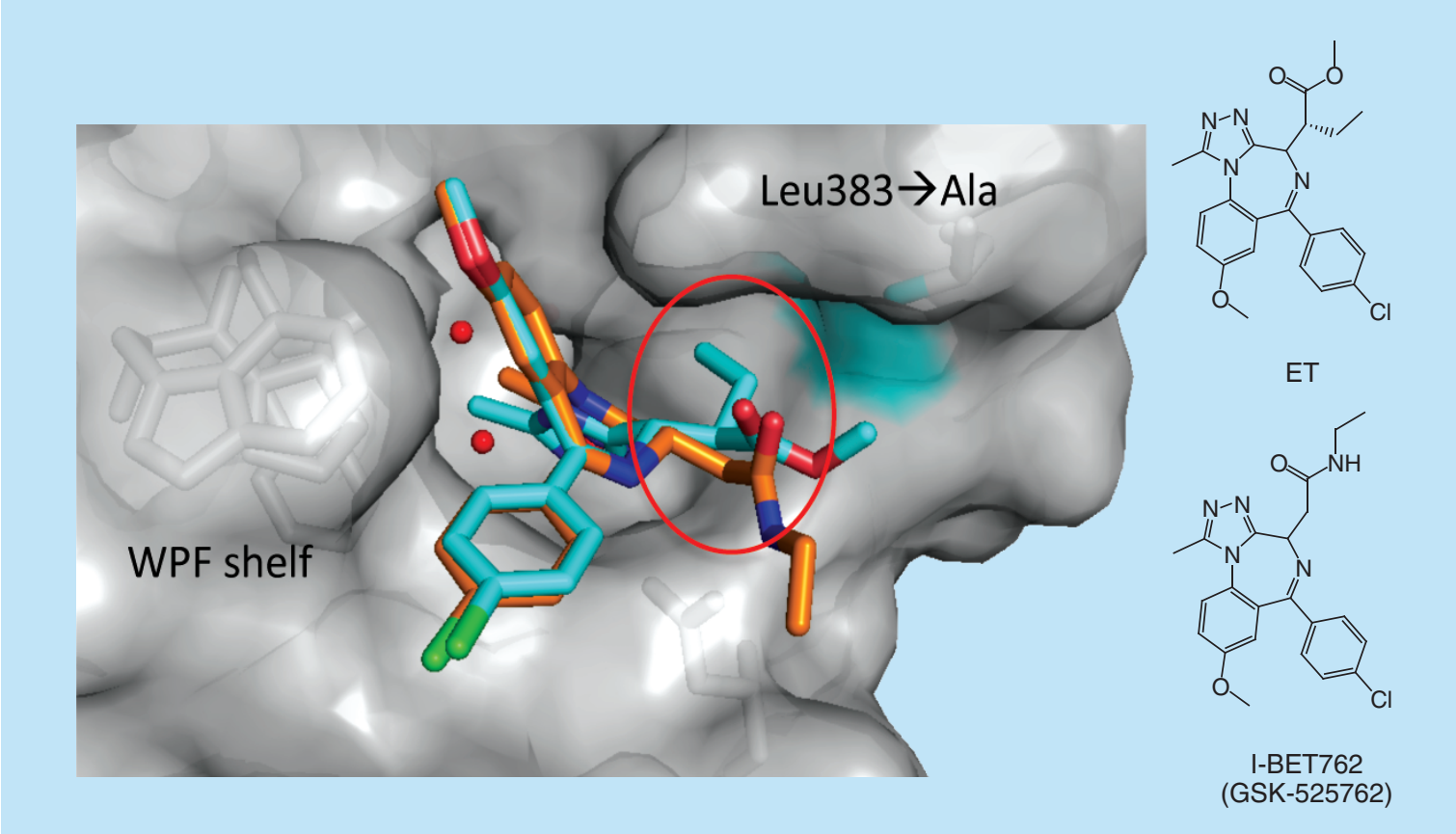

Figure 9. Bump-and-hole approach. Superposition of the $\mathrm{x}$-ray structure of the $\mathrm{ET}$ (in cyan) bound to the BRD2-BD2 1383 (in gray, $P D B$ BQEW) and parent ligand I-BET762 (in orange) bound to the BRD2-BD2 (not shown

served within BET bromodomains. Mutation of this into a whe group attempted to exploit mutations in into a smaller amino acid (Ala) created a 'hole' that did other conserved residues around the KAc binding site and structural integrity, leading to mutant BET pro- ever, these new mutations did not lead to the desired teins of reasonable functionlity that could accommo- increase in binding selectivity for mutan desired teins of reasonable functionality that could accommo-
date a modified inhibitoase in binding selectivity for mutant versus
wild-type when targeted with appropriately designed derivate (named ET, Figure 9) of a methylester analog of I-BET762 bumped (+)-JQ1/I-BET762 analogs. was designed and found to bind to all mutant BET bromodomains with nanomolar affinities, while only PROTAC approach

binding the wild-type versions with single- to double- With the aim to yield new chemical tools for studying digit micromolar affinities. ET achieved up to 540 -fold BET bromodomain protein, Zengerle et al. described selectivity and no less than 30-fold (average 160-fold) a new series of PROTACs (Proteolysis Targeting Chiacross the entire BET subfamily, and high selectivity mera) molecules to trigger the intracellular destruction was retained within tandem constructs. To validate of BET proteins [112]. PROTACs are hetero-bifuncthis strategy, $\mathrm{x}$-ray structures of $\mathrm{BRD} 2-\mathrm{BD} 2_{\mathrm{L} 338 \mathrm{~A}}$ in tional small molecules that allow selective recruitmen complex with the methylated and ethylated bumped of a protein of interest to the ubiquitin-proteasome proligands were solved (PDB 4QEV and 4QEW respec- teolytic machinery. By linking an optimized drug-like tively), showing that they adopt the same bind- ligand able to bind to the von Hippel-Lindau E3 ligase ing mode as I-BET 62 and (+)-JQ1, positioning the protein to the BET bromodomain inhibitor (+)-JQ1 respective methyl and ethyl substituents toward the researchers at the University of Dundee were able to hole introduced by the mutation (Figure 9). They went achieve rapid, effective and prolonged degradation of on and applied the approach in cells to demonstrate BET bromodomains with small molecules. Unexpect-

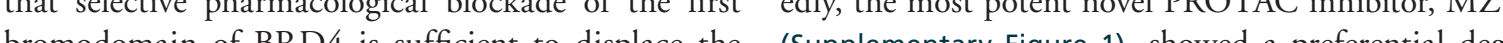
bromodomain of BRD4 is sufficient to displace the (Supplementary figure 1), showed a preferential degbinding of BRD4 being principally inflenced by BD1 BRD2 and BRD3 at relatively low co ly hor over BD2 [111. This study represents the fist devel- sectivity window transted in a more BRD4-specif opment of an allele-selective bump-and-hole approach downstrem transcriptional response in cancer cells against a protein-protein treated with MZ1 as compared with treatment with 

(+)-JQ1 [112]. The VHL-targeting BET PROTACs bio-JQ1 provided evidence that bio-JQ1 tends to co-
reported in this study demonstrate for the first time occupy enhancers where there are substantial BRD4 the possibility for turning unselective or pan-selective signals, and lower signals for BRD2 and BRD3. These inhibitors into chemical degraders of enhanced tar- results indicated that the pattern of (+)-JQ1 occupancy get selectivity profile. The authors speculated that the of chromatin is most strongly correlated with that of observed target selectivity could arise from preferential BRD4 in MM1.S cells [116].

recruitment of BRD4 relative to BRD2/3 by MZ1 in a ternary complex with the VHL E3 ligase, or more effcient downstream polyubiquination of lysine residues on the surface of BRD4, which warrant further structural and mechanistic studies. Overall, MZ1 showed improvements over (+)-JQ1 as a chemical probe due to its more limited transcriptional response and as a potential molecular therapeutic, providing unique opportunity to validate the therapeutic benefit of selec-
tive BRD4 removal, for example, compared with or in tive BRD4 removal, for example, compared
addition to pan-selective BET inhibition.

addition to pan-selective BET inhibition. A similar approach to degrade BET bromodo-
mains with a PROTAC molecule was pursued by $\mathrm{Lu}$ et al. [113]. In this case, OTX015 was used as the BET bromodomain binding ligand, which was joined to a phtalimide binding ligand for the E3 ligase (Supplementary Figure 1) induced effective degradation (Supplementary Figure 1) induced effective degradation anti-proliferative and $c$-MYC suppessive effect compared with (+)-JQ1 and OTX015 alone [113114]. Inteestingly no target degradation selectivity betwe BRD2, BRD3 and BRD4 was observed A simil PROTAC strategy was followed by Winter et al. where $(+)-J Q 1$ was conjugated to the same phtalimide ligand for cereblon, which induced cereblon-dependent BET protein degradation in cells and in vivo and delayed eukemia progression in mice [115]. Although the new compound dBET1 (Supplementary Figure 1) showed pan-selectivity for inducing degradation of BET proteins, no noteworthy intra-BET selectivity was observed [115].

\section{Chem-Seq approach}

The ability to map direct interactions of molecules with chromatin genome-wide could provide important information on the on-target effect of small molecules aiding biological insights. Anders et al. used Chem-seq, allet unbiased DNA sequencing, to investigate the allel unbiased DNA sequencing, to investigate the (+)-JQ1 to the BRD2, BRD3 and BRD4 in multiple myeloma cells (MM1.S) [116]. The authors showed that the genomic sites bound by a biotinylated derivate of (+)-JQ1 (bio-JQ1, Supplementary Figure 1) are highly similar to the sites occupied by the native BRD2 BRD3 and BRD4 proteins. However, inspection of gene tracks for regions differentially occupied by

\section{Off-target effects}

ain/kinase inhibitors

The target selectivity and potential off-target effects

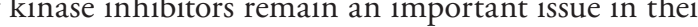
tilization as kinase chemical probes [117]. Several

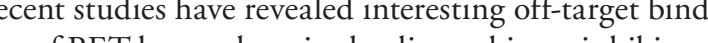
in Intrigued by the observation that BRD4 exerts a de a decided to elucidate the $x$-ray structure of the potent CDK BRDT-BD1 (PDB 4KCX, rigure 10) bromodomain (BRDT-BDI (PDB 4KCX, figure 10) [119]. Surprisingly the BRDT-BD1 of $37 \mu \mathrm{M}$ in $\mathrm{PPCR}-\mathrm{wased}$ asay). The pridine oxide moiety of dinaciclib acts as a KAc The pyrinteracting with the crucial Asn amio aid (N109 in BRDTBD1) and the pyrazopyrimid (N109. ety lies parallel to the WPF shelf, making additional water-mediated hydrogen bond with the backbone of ZA channel residues P55 and V56. An additiona profiling of dinaciclib against a panel of 24 bromodomains revealed that apart from BET bromodomains, for dinaciclib were TAF1 and TAF1L. These finding were consistent with the hypothesis that BET protein could be potential off-targets of ATP-site-directed kinase inhibitors. Because of the potential of synergisic pharmacology between kinase and BET inhibition in cancer cell lines, several kinase inhibitos have since been investigated for their potential to bind to BET Through a robotic co-crystallization screening campaign Ember et al. demonstrated that a number of protein kinase inhibitors currently in the clinic, for example, BI-2536 (Supplementary Figure 1) and G-101348 (Supplementary Figure 1), a PLK1 and JAK2-FLT3 (supp and secering chan 14 aRD 1 inibtors, the majoity of them showing microm tion of BET proteins $x$ - say cocystal structures showed hat most of he kinase inhipers tak a lighly diffeKAc bing ring sine of BRDT, albet with a differimal binding affinity compared with (+)-JQ1 $(K$ for the only other bromodomains with binding potential

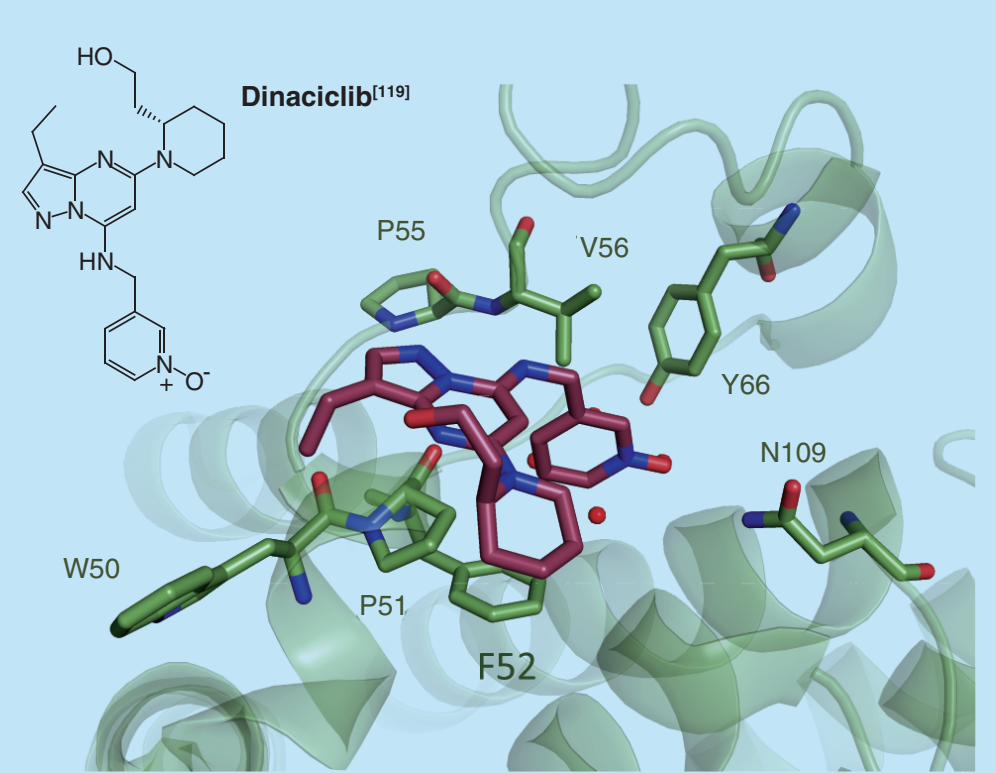
Figure 10. Dual bromodomain/kinase inhibitors. X-ray structure of the dinaciclib (in magenta) bound to the
BRDT-BD1 BET bromodomain (in green, PDB 4KCX).

ent conformation when they bind to the bromodomai compared with when they bind to the ATP site of the states, and consequently reduced binding potengy toward BET bromodomains. Importantly, none of the kinase inhibitors investigated in the paper interact with the characteristic water network of the KAc binding site, opening opportunities for the development of a novel class of BET bromodomains, directly interacting with the residues of the binding site. In parallel, Dittman et al. following a quantitative chemoproteomic approach demostrated that LY294002 (Supplementary Figure 1) and its negative control ana$\log$ LY303511 (Supplementary Figure 1), two inhibitors of PI3K enzymes, were both able to block the first, bu not the second, bromodomain of BRD2, BRD3 an BRD4 [120]. Further, it was found that the cellular and transcriptional effects of LY303511 were accounte for largely by their off-target interaction with BET bromodomains.

In a separate study, Ciceri et al. also reported that several clinical kinase inhibitors, such as BI-2536 an TG-101348 (Supplementary Figure 1) inhibit bromodomains with therapeutically relevant potencies $[122]$ In fact, TLK1 and its infition B-2536 has been the focus of sone of the effors to develop dual inhibilent oncogenic pathways exemplify a new stregfor ration ing and provide a new structural framework for the rition dual-activity BET-kinas inhibiots.

GABA inhibitors

For many years, the benzodiazepine chemical group has been considered as a 'privileged scaffold' in drug dis.125. A large number of approved inhibmoiety. In addition, several bromodomain inhibitors in clinical trials such as I-BET762, (+)-JQ1 and OTX15 (Figure 1) also have a benzodiazepine-based structure. Filippakopoulos et al. provided insights into the structure-activity relationships and selectivity of the approved GABA inhibitors toward the BET bromodomains $[126$. They found that alprazolam (Figure 11) inds with low micromolar affinity to BRD4-BD1. The co-crystal structure of alprazolam with BRD4BDI showed that the triazolo ring of alprazolan fors a hydrogen bond with the conserved Asn residue. Unexpectedly, also midazolam interacts with BeT bromodomains, preferentially with $\mathrm{BD} 2$, in the iemar range of affinity. Co-crystal structure of dazolam with BRD4-BDI shows that although ine triacks the hydrogen bond forming nitrogen the triazolo group, it is able to bind to this BEI conser ite [126] Hower the minside of hac Kand compour ide effects de to inhibion of BET bo

\section{Conclusion \& future perspective}

Epigenetic control of gene expression plays a significant of including cancer and inflammation. Protein readers of histone acetylation and ther 


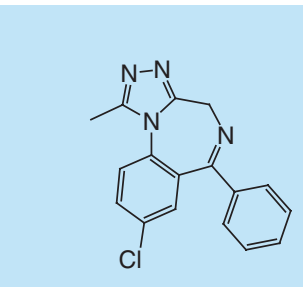

Alprazolam

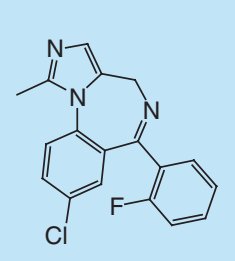

Figure 11. GABA inhibitors. Chemical structure

bromodomains have emerged as attractive drug targets for these diseases, yet little is known about their indvidual biological function, how they work inside cells in how they should be best modulated pharmacologically. For these reasons, much interest has spurred in epigene-
tic medicinal chemistry and drug discovery, and speciftic medicinal chemistry and drug discovery, and specifiprobes for bromodomains, to help fill these knowledge gaps and validate new potential drug targets.

In this review, we have highlighted the state-of-
gaps and validate new potential drug targets. he-art in the field, focusing on the in vitro on-target selectivity of the probes reported to date, as meare using biophysical techniques mostly against puri- $c$ fed proteins. Robust in vitro selectivity profling of chemical probes is advocated, using appropriate assays such as the BromoScan assay provided by DiscoveR and related assays over less quantitative methods, for key target classes, for example, kinases and GPCRs. We expect efforts in future to focus on assessment of target engagement selectivity inside cells in an unbiased fashion. Progress in this direction will likely take advantage of new developments in molecular biology and genome editing, for example, by clustered regularly interspaced short palindromic repeats (CRISPR)Cas9 [127], and the translation of biophysical measurements directly in cellular environment, as exemplified by cellular thermal shift assay (CETSA) [128], cellular thermal profiling by mass spectrometry [129] and methods using bioluminescence resonance energy transfer BRET) such as NanoBRET assay [130,131].

The cutting-edge progress achieved in recent years in the field by both industrial and academic scientists demonstrates that it is possible to obtain some degree f target selectivity within selected bromodomain subily members selectivity aginst BET bromodomains, Particularly BRD4-BD1, is con ment to eliminate any unwant BRD4 dependent cellular effect. It has nevertheless remained a challenge to achieve intr fomily single target selectivity. When this vided find both physiological and pathophysiological contexts, and example, DSF, as well as selectivity profiling against

cannot be achieved by targeting the KAc pocket, alternare specific PPI binding sites outside the KAc pocket will likely need to be explored It is also envisaged that covalent reversible or irreversible inhibition, which has so far not been much exploited domains, could help to enhance on-target selectivity.

In addition to conventional inhibitors, more sophisticated chemical biology approaches such as bump-and-hole and PROTACs have demonstrated that they can lead to single-target selectivity even when starting from the pan-selective inhibitor (+)-JQ1. Suc new more refined chemical tools are posed for many pplications in the near future to dissect individua physiological roles of BET proteins in vivo, and could be widely extended to other BCPs as well as other pigenetic reader domains.

Despite the extraordinary progress to date, there still emain many challenges facing the field ahead. Bromodomains have proven to be highly ligandable' targets, epecially for snall tou o dent on the nature of each target, yet inhibitor poten can be achieved with most bromodomains. From a drug discovery perspective, modulation of BET proteins with romodomin inhibitors is now a validated therapeuic approach. It remains to be seen to what extent the many inhibitors for non-BET bromodomains that are available and will continue to emerge can exert a desired evel of cellular efficacy, and consequently the question of which target will prove to be 'druggable' as well as 'ligandable' remains open. Validated drug targets will likely be determined more by the biology of individual $\mathrm{BCPs}$, than by the ligandability of their bromodomain. Effective coupling of chemical with biological target validation approaches, surmounting inherent challenges socitited with the complexity of many BCPs and their

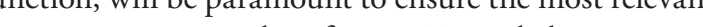
de do cientife scientific community
biological questions.

\section{supplementary data}

view the supplementary data that accompany this paper Tere visit the journal website at: www future-science com oilfull/10.4155/fmc-2016-0059

inancial \& competing interests disclosure The authors are grateful to the organizations that have pro-

ty of Dundee, including the European Research Council (ERC- tions or financial involvement with any organization or entity iy of Dundee, including the European Research Council (ERC- tions or financial involvement with any organization or entity (ove Council (BBSRC, grant BB/J001201/2 and David Phillips Fel- those disclosed. lowship BB/G023123/2 to A Ciulli). C Galdeano thanks the European Commission PIEF-GA-2012-328030 (Marie-Curie Intra-European Fellowship), and the Secretaria d'Universitats

Generalitat de Catalunnya and the $7^{\text {th }}$ European Framework This article is distributed under the terms of the Creative Program-Marie Curie COFUND (contract 600385; Beatriu de Commons Attribution License 4.0 which permits any use, Pinos Fellowship). Biophysics and drug discovery activities at distribution, and reproduction in any medium, provided the Dundee are supported by a Wellcome Trust strategic award original author(s) and the source are credited. To view a copy 100476/Z/12/Z to the Division of Biological Chemistry and of the license, visit http://creativecommons.org/licenses/ Drug Discovery. The authors have no other relevant affilia- by/4.0/

\section{Executive summary}

- With the publication in 2010 of BET inhibitors (+)-JQ1 and I-BET762, there has been an explosion of small molecules discovered or designed to target bromodomains in the human family.

- The high similarity within the different subfamilies of bromodomains, in particular at the level of their
KAc binding sites, poses inherent challenges to the development of selective bromodomain inhibitors for delivering high-quality chemical probes.

- Despite achieving several compounds in clinical trials, the development of BET inhibitors as chemical probes of individual BET proteins has remained a major challenge, due to the lack of single target selectivity.

- We exemplify the efforts to obtain selective bromodomains-class inhibitors with case studies taken not only from the BET subfamily but also from bromodomain targets in other subfamilies.

- The ability of BEF inhiber individually in the control of gene transcription in chromatin.

- Innovative chemical biology and chemical genetic approaches such as bump-and-hole, PROTACs and Chem-Seq approaches allow to address and enhance on-target selectivity and efficacy, and interrogate context-dependent mode of action of BET inhibitors.

- Several studies have revealed interesting and unexpected off-target binding of BET bromodomains by - inhibitors previously developed against other targets, such as protein kinases and receptors. - chemical probes, of the quality necessary for use as tools to address biological auestions highly selective

approaches including assessment of target engagement selectivity in bromodomains inside cells in an unbiased fashion; covalent reversible or irreversible inhibitor design; development and application of sophisticated chemical biology approaches to enhance chemical probing; and finally, closer synergy between semical and biological target validation.

\section{References}

Papers of special note have been highlighted ar:

Goldberg AD, Allis CD, Bernstein E. Epigenetics: a

Strahl BD, Allis CD. The language of covalent histon

fications. Nature 403(6765), 41-45 (2000).

Jenuwein T, Allis CD. Translating the histone code.

Kouzarides T. Chromatin modifications and their
function. Cell 128(4), 693-705 (2007)

Arrowsmith CH, Bountra C, Fish PV, Lee K, Schapira M. Epigenetic protein families: a new frontier for drug discovery. Nat. Rev. Drug Discov. 11(5), 384-400 (2012).
Patel DJ, Wang Z. Readout of epigenetic modification Annu. Rev. Biochem. 82(1), 81-1. Feinberg AP, Vogelstein B. Hypomethylation distinguishes genes of some human cancers from their normal
counterparts. Nature 3011(5895), 89-92 (1983).

Feinberg AP, Tycko B. The history of cancer epigenetics. Nat Rev. Cancer 4 (2), 143-153 (2004).

Dawson MA, Kouzarides T. Cancer epigenetics: from

Kouzarides T. Acetylation: a regulatory modification to rival Kouzarides T. Acetylation: a regulatory modification
phosphorylation? $E M B O J .19(6), 1176-1179(2000)$.

Yang X-J, Seto E. Lysine acetylation: codified crosstalk
with other posttranslational modifications. Mol. Cell $31(4)$ 449-461 (2008) 
12 Kuo $\mathrm{MH}$, Allis $\mathrm{CD}$. Roles of histone acetyltransferases and deacetyl
(1998).

13 Yang X-J, Seto E. HATs and HDACs: from structure, function and regulation to novel strategies for therap

prevention. Ong

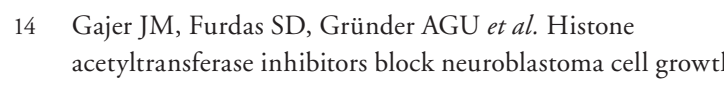
in vivo. Oncogenessis 4, e137 (2015).

15 Falkenberg KJ, Johnstone RW. Histone deacetylases and their inhibitors in cancer, neurological diseases and imm
disorders. Nat. Rev. Drug Discov. 13(9), 673-691 (2014). 16 Marks P, Rifkind RA, Richon VM, Breslow R, Miller T, Kelly WK. Histone deacetylases and cancer: causes and therapies. Nat. Rev. Cancer 13), 194-202 (2001).

7 Filippakopoulos P, Qi J, Picaud Set al. Selective inhibition of BET bromodomains. Nature 468(7327), 1067-1073 (2010).

18 Nicodeme E, Jeffrey KL, Schaefer U et al. Suppression
of inflammation by a syntheric histone mimic. Nature of inflammation by a synthetic

19 Philpott M, Yang J, Tumber T et al. Bromodomain-peptide displacement assays for interactome mapping and
discovery. Mol. BioSyst. $7(10), 2899-2908$ (2011).

20 Huston A, Arrowsmith CH, Knapp S, Schapira M. Probing Arrowsmith CH, Audia JE Ausin Co peril of chemical probes. Nat. Chem. Biol. 11(8), 536-541 (2015).

- Timely viewpoint advocating the scientific community elevate level of rigorous development and application of chemical probes in research. Describes the launch of the 'Chemical Probes Portal' as an online community resource committed to serve these purposes and to promote wide Me

22 Morinière J, Rousseaux S, Steuerwald U et all. Cooperative binding of two acetylation marks on a histone tail by
bromodomain. Nature 461 (7264), $664-668$ (2009).

23 Sanchez R, Meslamani J, Zhou M-M. The bromodomain: from epigenome reader to druggable target. Biochim. Biophys

Filippakopoulos P, Picaud S, Mangos M et al. Histone recognition and large-scale structural analysis of the $h$

Mujtaba S, He Y, Zeng L et al. Structural basis of lysineacetylated HIV-1 Tat recognition by PCAF bromodoma

26 Plotnikov AN, Yang S, Zhou TJ, Rusinova E, Frasca A,

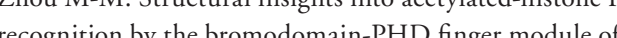
353-360 (2014).

27 Mujtaba S, He Y, Zeng L et al. Structural mechanism of the bromodomain of the coactivator CBP in $\mathrm{p} 53$ transcription

activation. Mol. Cell 13(2), 251-263 (2004).

28 Zeng L, Zhou M-M. Bromodomain: an acetyl-lysine binding domain. FEBS Lett. 513(1), 124-128 (2002)
29 Mujiaba S, Zeng L, Zhou MM. Structure and acetylysine recognition of the bromodomain. Oncogene $26(37)$

30 Marchand J-R, Cafilisch A. Binding mode of acetylated ChemMed Chem 10(8), 1327-1333 (2015).

31 Dhalluin C, Carlson JE, Zeng L, He C, Aggarwal AK, Zhou bromodomain. Nature 399 (6735), 491-496 (1999)-

Romero FA Talor AM, Crawfod TD, Tai V, CoreA,

Magnuson S. Disrupting acetyl-lysine recogyit cote A, Magnuson S. Dissupting acetyl-lysine recognition: progress
in the development of bromodomain inhibitors. J. Med. Chem. 59(4), 1271-1298 (2016).

33 Fu L-L, Tian M, Li X et al. Inhibition of BET bromodomains as a therapeutic strategy for cancer dru

4 Shi J, Vakoc CR. The mechanisms behind the therapeutic activity of BET brom 736 (2014).

35 Wu S-Y, Chiang C-M. The double bromodomain-containing chromatin adaptor Brd4 and transcriptional regulation.
J. Biol. Chem. 282 (18), 13141-13145 (2007).

36 Chiang C-M. Brd 4 engagement from chromatin targeting to transcriptional regulation: selective contact with acety
histone H3 and H4. FIO0O Biol. Rep. 1, 98 (2009).

37 Gamsjaeger R, Webb SR, Lamonica JM, Billin A, Blobel GA, Mackay JP. Structural basis and specificity of acetylated transcription factor GATA1 recognition by BET family bromodomain protein
$2632-2640$ (2011).

38 LeRoy G, Rickards B, Flint SJ. The double bromodomain proteins Brd2 and Brd3 couple histone acetylation

39 Gallenkamp D, Gelato KA, Haendler B, Weinmann $\mathrm{H}$. Bromodomains and their pharmacologial in

40 Albrecht BK, Gehling VS, Hewitt MC et al. Identification of a benzoisoxazoloazepine inhibitor (CPI-0610) of the bromodomain and extra-terminal (BET) family as a
candidate for human clinical trials. J. Med. Chem. 59 (4), candidate for human
$1330-1339(2016)$.

41 Frye SV. The art of the chemical probe. Nat. Chem. Biol.

2 Bunnage ME, Gilbert AM, Jones LH, Hett EC. Know your target, know your molecule. Nat. Chem. Biol. 11(6), 368-372 (2015).

3 Chemical Probes.

44 Fedorov $\mathrm{O}$, Lingard $\mathrm{H}$, Wells $\mathrm{C}$ et al. $[1,2,4]$ triazolo $[4,3-d$ Chem. 57(2), $462-476$ (2014)

Bunnage ME, Chekler ELP, Jones LH. Target validation using ch,
(2013).

46 Cohen P. Guidelines for the effective use of chemical ir roles in cell regulation. Biochem J. 425(1), 53-54 (2010)
47 Workman P, Collins I. Probing the probes: fitness factors for small molecules tools. Chem. Biol. 17(6), 561-577

48 Demont EH, Bamborough P, Chung C-W et al. 1,3-dimethyl benzimidazolones are potent, selective
inhibitiors of the BRPF1 bromodomain. ACS Med. Chem Inhtibitors of the BRPF brons $1190-1105$ (2014)

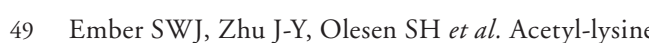
binding site of bromodomain-containing protein 4 (BRD4) interacts with diverse kinase inhibitors. ACS Chem. Biol. 9(5), 1160-1171 (2014).

50 Jennings LE, Measures AR, Wilson BG, Conway S. Phenotypic screening and fragment-based approaches to the discovery of small-molecule bromodomain ligands. Future

51 Vidler LR, Brown N, Knapp S, Hoelder S. Druggability

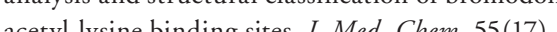
acety-lysine binding

52 Brand M, Measures AM, Wilson BG et al. Small molecule inhibitors of bromodomain-acety
Chem. Biol. $10(1), 22-39(2015)$

53 Filippakopoulos P, Knapp S. Targeting bromodor epigenetic readers of yysine acetylation. Nat. Rev. Drug Discov. 13(5), 337-356 (2014)

54 Chung C-W, Coste H, White JH et al. Discovery and characterization of small molecule inhibitors of the BET family bromoder of $J$

55 Fish PV, Filippakopoulos P, Bish G et al. Identification of a chemical probe for bromo and extra C-terminal bromodomain inhibition through optimization of a
fragment-derived hit $J$ Med Chem 55(22), 9831-9837 (2012).

56 Gosmini R, Nguyen VL, Toum J et al. The discovery of 1-BET/26 (GSK1324/26A), a potent terahydroquino ApoA1 up-regulator and selective BET bromodomain

Lucas X, Wohlwend D, Hugle M et al. 4-Acyl pyrroles. Angew. Chem. Int. Ed. Engl 52 (52), 14055-14059 (2013).

58 Hugle M, Lucas X, Weitzel G et al. 4-Acyl pyrrole derivatives yield novel vectors for designing inhibitors
of the acetyl-lysine recognition site of BRD4 (1). J. Med. Chem. 59(4), 1518-1530 (2016)

59 Hewings DS, Wang M, Philpott M et al. 3,5-dimerhylisoxazoles act as acetyl-yysine-mimetic bromodomain ligand

60 Hewings DS, Fedorov O, Filippakopoulos P et al. bromodomain ligands. J. Med . Chem. $56(8), 3217-3227$ (2013).

61 Ran X, Zhao Y, Liu L et all Structure-based design of gamma-carboline analogues as potent and specific BET bromo
62 McKeown MR, Shaw DL, Fu $\mathrm{H}$ et al. Biased multicomponen reactions to develop novel bromod
Chem. $57(21), 9019-9027$ (2014). Rooney TPC, Filippakopoulos P, Fedorov $\mathrm{C}$ tall. A series of
potent CREBPP bromodomain ligands reveals an inducedffit pocket stabilized by a cation-pi interaction. Angew. Chem. Int. Ed. Engl 53(24),

A beautiful example of academic ligand design projec starting small from a fragment to optimize specific interactions and achieve potency and selectivity against a challenging bromodomain target.

64 Hay DA, Fedorov O, Martin Setal. Discovery and optimization of small-molecule ligands for the $\mathrm{CBP} / \mathrm{p} 300$ bromodomains. J. Am. Chem. Soc. 136(26), 9308-9319 (2014)

65 Sachchidanand, Resnick-Silverman L, Yan Set al. Target structure-based discovery of small molecules that block human
p53 and CREB binding protein association. Chem. Biol. 13 (1) $81-90$ (2006).

66 Pan C, Mezei M, Muitaba Set al. Structure-guided immunodeficiency virus 1 Tat association with the hw coactivator P300/CREB binding protein-associated factor. J. Med. Chem. 50(10), 2285-2288 (2007).

67 Borah JC, Mujtaba S, Karakikes I et al. A small molecule binding to the coactivator CREB-binding protein blocks apoptosis in cardionyocyes. Chem. Bid. 18(4), 531-541

68 Hewings DS, Rooney TPC, Jennings LE et al. Progres in the development and application of small molecule
inhibitiors of bromodomain-aceryl-ysine interactions. $J . M e$ Chem. 55 (22), 9393-9413 (2012).

69 Gerona-Navarro G, Yoel-Rodriguez, Muitaba S et al. Rational design of cyclic peptide modulators of the transcriptional coactivator CBP. a new class of ps3 inhibitiors. . Am. Che

70 Hammitzsch A, Tallant C, Fedorov O et al. CBP30, a selective

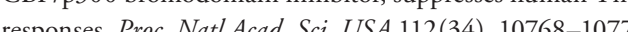
(2015).

71 Picaud S, Fedorov O, Thanasopoulou A et al. Generation of a selective small molecule inhibitor of the CBP/ 3300 $5106-511$ (2015).

2 Unzue A, Xu M, Dong J et al. Fragment-based design of selective nanomolar ligands of the CREBBP bromodomain.

73 Xu M, Unzue A, Dong J, Spiliotopoulos D, Nevado C, by high-throughput docking and hit optimization guided by molecular dynamics. J. Med. Chem. 59(4), 1340-1349 (2016).

74 Flynn EM, Huang OW, Poy Fet al. A subset of human bromodomains recognizes butyryllysine and crotonyllysine
histone peptide modifications. Structure 23(10), 1801-181 (2015). Clark PGK, Vieira LCC, Tallant Cet al. LP99: discovery
and synthesis of the first selective BRD7/9 bromodomain 
(2015)

6 Theodoulou NH, Bamborough P, Bannister AJ et al. Discovery of I-BRD 9 , a selective cell active chemical prob
for bromodomain containing protein 9 inhibibion. $J$. Chem. 59 (4), 1425 -1439 (2016).

77 Hay DA, Rogers CM, Fedorov O et al. Design and and BRDO bromodomins Med Chem Commun 1381-1386 (2015

78 Chen $\mathrm{P}$, Chaikuad A, Bamborough $\mathrm{P}$ et al. Discovery probe for the bromodomains BAZ2A and BAZ2B. J. M Chem. 59(4), 1410-1424 (2016).

79 Martin LJ, Koegl M, Bader G et al. Structure-based design of an in vivo active selective BRD9 inhibitor. J. Med. Chem. doi:10.1021/acs.jmedchem.5b01865 (2016) (Epub ahea

- Exemplary medicinal chemistry journey leading to most high-quality chemical probes for BRD9 bromodomain library primary screen in parallel by three ofthogn biophysical techniques, hit validation and careful structure-based optimization of potency and selectivity versus brd4-BD1 off-target.

80 Sun H, Liu J, Zhang J et al. Solution structure of BRD7 bromodomain and its interaction with acetylated peptides from histone $\mathrm{H} 3$ and $\mathrm{H} 4$. Biochem. Biophys. Re

Picaud S, Strocchia M, Terracciano Set al. 9 H-purine scaffold reveals induced-fit pocket plasticity of the BRD
bromodomain. J. Med. Chem. $58(6), 2718-2736$ (2015).

Tallant C, Valentini E, Fedorov Oetal. Molecular basis of histone tail recognition by human TIPS PHD finger and bromodomain of the chromatin remodeling complex NoRC. Structure 23(1), 80-92 (2015)

83 Ferguson FM, Fedorov O, Chaikuad A et al. Targeting lowdruggabilitit bromodomains: fragment based screening and inhibitor design against the BAZ2B

84 Drouin L, McGrath S, Vidler LR et al. Structure enabled design of BAZ2-ICR, a chemical probe targeting the $58(5), 2553-2559$ (2015).

85 Sharp PP, Garnier J-M, Huang DCS, Burns CJ. Evaluation of functional groups as acetyl-lysine mimetics for BET bromodomain inhibition. Med. Chem. Commun

86 Chung C-W, Dean AW, Woolven JM, Bamborough P.

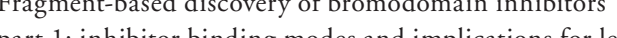
part 1: inhibitor binding modes and implications
discovery. J. Med. Chem. $55(2), 576-586$ (2012).

First report of successful application of full library biophysical fragment screening against a brory . biophysical fragment screening against a bromodoma
target. The authors identified diverse chemotypes to probe hot spot interactions at the target Kac binding site, providing many attractive starting points for chemical elaboration and proof-of-concept of the ligandability of brom

www.thesgc.ors

88 Palmer WS, Poncet-Montange G, Liu G et al. Structureguided design of $1 A C S-9571$, a selective high-affinity dual TRIM24-BRPFi bromodomain

89 Bennett J, Fedorov O, Tallant C et al. Discovery of a of TRIM24 and BRPF. J. Med. Chem. 59 (4), 1642-1647 (2016).

90 Harner MJ, Chauder BA, Phan J, Fesik SW. Fragmentbased screening of the bromodomai
Chem. 57 (22), 9687-9692 (2014).

91 Chaikuad A, Petros AM, Fedorov O, Xu J, Knapp S. Structure-based approaches toward identification of fragments for the low-druggability ATAD2 bromodoma th

92 Bamborough P, Chung C-W, Furze RC et al. StructureATAD2 bromodomain inhibitors. I. Med. Phem. $58(18)$ 6151-6178 (2015)

- Together with its next-of-kin back-to-back article [93], provides a beautifully described med chem story from the GSK team to successfully target the low-druggability ATAD2 bromodomain. A textbook example of how structure-guided drug design is best conducted, trackin consistently all key parameters of chemical probe development - from SAR, selectivity vs off-target, to compound optimization in a logical manner.

93 Demont EH, Chung C-W, Furze RC et al. Fragmentbased discovery of low-micromolar ATAD2 bromodomain
inhibitors. J. Med. Chem. 58(14), 5649-5673 (2015).

94 Urick AK, Hawk LML, Cassel MK et al. Dual screening
of BPTF and Brd4 using protein-observed fluorine NMR uncovers new bromodomain probe molecules. ACS Chem

Biol. 10(10), 2246-2256 (2015).

95 Vangamudi B, Paul TA, Shah PK et al. The SMARCA2/4 ATPase domain surpasses the bromodomain as a drug target in SWIISNF-mutant cancers: insights from CDNA rescue (2015).

96 Nakamura Y, Umehara T, Nakano Ket al. Crystal structure of the human $\mathrm{BRD} 2$ bromodomain: insights into J. Biol. Chem. 282(6), 4193-4201 (2007).

97 Gacias M, Gerona-Navarro G, Plotnikov AN et al. Selective chemical modulation of gene transcription favors
oligodendrocyte lineage progression. Chem. Biol. $21(7)$, oligodendrocyte linag
$841-854$ (2014).

98 Chiang C-M. Nonequivalent response to bromodomainargeting BET inhibitors in oligodendrocyte cell
decision. Chem. Biol. 21(7), 804-806 (2014).

9 Lamonica JM, Deng W, Kadauke Set tal. Bromodomain
protein Brd3 associates with acetylated GATA1 to promote Acad. Sci. USA 108 (22), E159-E168 (2011).

100 Shi J, Wang Y, Zeng L et al. Dissupting the interaction of BRD4 with diacetylated Twist suppresses tumorigenesis in
basal-like breast cancer. Cancer Cell 25(2), 210-225 (2014)

101 Zhang G, Plotrnikov AN, Rusinova E et al. Structure-guided design of potent diazobenzene inhibitors for the BET

102 McLure KG, Gesner EM, Tsujikawa L et al. RVX-208, an inducer of ApoA-I in humans, is a BET bro
antagonist. PLOS ONE $8(12)$, e83190 (2013).

This paper and another parallel study [103] describe comprehensive structural, biophysical and cellular characterization as chemical probe of Resverlogix compo Rvx-208 - one of the first reported BD2-selective BET

inhibitor.

103 Picaud S, Wells C, Felletar I et al. RVX-208, an inhibitor of BET transcriptional regulators with selectivity for the second bromodomain. Proc. Nall Acad. Sci. USA 110(49),

104 Baud MGJ, Lin-Shiao E, Zengerle M, Tallant C, Ciulli A. expanding the scope of the bump-and-hole approach for selective bromo and extra-terminal (BET) bromodom in inhibition. J. Med. Chem. S9(4), 1492-1500 (2016).

105 Zenith epigenetics

www.zenithepigenetics.com

106 Raux B, Voitovich Y, Derviaux Cetal. Exploring selective inhibition of the first bromodomain of the human bromodomain and extra-terminal domain (BET) proteins

107 Lejeune P, Sugawara T, Gelato KA et al. BAY 1238097, a tumor models. [abstracts. In: Proccedings of the 106 th A A m Meeting of the American Association for Cancer Ressarch; Cancer Res. 75(Suppl. 15), Abstract nr3524 (2015).

108 Coude M-M, Braun T, Berrou J et al. BET inhibitor OTX015 targets BRD2 and BRD4 and decreases $\mathrm{C}$-MYC in acute
leukemia cells. Oncotarget 6 (19), $17698-17712$ (2015).

109 Shah K, Liu Y, Deirmengian C, Shokat KM. Engineering unnatural nucleotide specificity for Rous sarcoma virus tyrosine kinase to uniquely label its direct
Acad. Sci. USA 94 $(8), 3565-3570$ (1997).

110 Bishop AC, Ubersax JA, Petsch DT et al. A chemical switch for inhibitior-sensitive alleles of
Nature $407(6802)$, 395-401 (2000).

111 Baud MGJ, Lin-Shiao E, Cardote Tetal Chemical biologe A bump-and-hole approach to engineer controlled selectivity of BET bromodomain chemical probes. Science 346(6209)

- Successful development of the bump-and-hole approach to achieve controlled probing of individual BET bromod
using a small molecule and to tease out the relative importance of BD1 and $\mathrm{BD} 2$ in the binding of Brd4 to

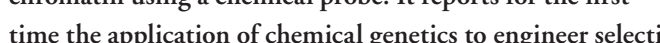
small molecule disruption of a potin-protein interaction.
12 Zengerle M, Chan K-H, Ciulli A. Selective small molecule induced degradation of the $\mathrm{BET}$ bromodomain protein $\mathrm{BRD}$ ACS Chem. Biol. 10(8), 1770-1777 (2015).

113 Lu J, Qian Y, Altieri M et al. Hijacking the E3 ubiquitin ligase cereblon to efficis
$22(6), 755-763(2015)$

114 Delmore JE, Issa GC, Lemieux ME et al. BET c-Myc. Cell 146(6), 904-917 (2011)

115 Winter GE, Burley DL Paulk ot al Phtalinite conjugation as a strategy for in vivo target protein
degradation. Science $348(6241), 1376-1381$ (2015).

16 Anders L, Guenther MG, Q1 et al. Genome-wide localization of small molecules. Nat. Biotechnol. 32(1), 92-96 (2013).

117 Hantschel O. Unexpected off-targets and paradoxical pathway activation by kinase inhibitors. ACS Chem. Bial.

118 Devaiah BN, Lewis BA, Cherman N et al. BRD 4 is an atypical kinase that phosphorylates serine2 of the RNA Sci. USA 109 (18), 6927-6932 (2012).

19 Martin MP, Olesen SH, Georg GI Schonbrunn E. Cyclindependent kinase inhibitor dinaciclib interacts with the acetyl-lysine recognition site of bromodomains. ACS Chem Biol. 8(11), 2360-2365 (2013).

120 Dittmann A, Werner T, Chung C-W et al. The commonly used PI3-kinase probe LY294002 is an inhibitor of BET

21 Zhang G, Smith SG, Zhou M-M. Discovery of chemical inhibitors of human b.
$11625-11668$ (2015).

- Landmark recent review of chemistry focus that provides a historical perspective on the discovery of bromodomain field.

122 Ciceri P, Müller S, O'Mahony A et al. Dual kinasebromodomain inhibitors for rationally designed
polypharmacology. Nat. Chem. Biol. $10(4), 305-312$ (2014).

123 Chen L, Yap JL, Yoshioka M et al. BRD4 structure-activity relationships of dual PLK1 kinase/BRD4 bromodomain
inhibitor BI-2536. ACS Med. Chem. Lett. 6(7), 764-769 inhibitor
(2015).

124 Welsch ME, Snyder SA, Stockwell BR. Privileged scaffolds Bio. $14(3), 347-361$ (2010).

125 Smith SG, Sanchez R, Zhou M-M. Privileged diazepine compounds and their emergence as bromodoma
inhibitors. Chem. Biol. 21(5), 573-583 (2014).

26 Filippakopoulos P, Picaud S, Fedorov $\mathrm{O}$ et al.

Benzodiazepines and benzotriazepines as protein interactio Binhibitors targeting bromodomains of the BET farty-

27 Doudna JA, Charpentier E. Genome editing. The new frontier of genome engineering with CRISPR-Cas
Science $346(6213)$. 1258096-1258096 (2014). 
128 Savitski MM, Reinhard FBM, Franken H et al. Proteomics. Tracking cancer drugs in living cells by thermal profiling of the proteome. Science 346(6205), 1255784 (2014).

129 Martinez Molina D, Jafari R, Ignatushchenko M et al. Monitoring drug target engagement in cells and tissues using the cellular thermal shift assay. Science 341(6141), 84-87 (2013).
130 Robers MB, Dart ML, Woodroofe CC et al. Target engagement and drug residence time can be observed in living cells with BRET. Nat. Commun. 6, 10091 (2015).

131 Machleidt T, Woodroofe CC, Schwinn MK et al. NanoBRET - a novel BRET platform for the analysis of protein-protein interactions. ACS Chem. Biol. 10(8), 1797-1804 (2015). 